\title{
Metric curvatures and their applications I
}

\author{
EMIL SAUCAN*
}

\begin{abstract}
We present, in a natural, developmental manner, the main types of metric curvatures and investigate their relationship with the notions of Hausdorff and Gromov-Hausdorff distances, which by now have been widely adopted in the fields of Imaging, Vision and Graphics. In addition we present a number of applications to the fields above, as well as to Communication Networks and Regge Calculus. Further connections with such established notions as excess and fatness are also investigated. While the present paper represents essentially a survey, a number of possible applications are presented here for the first time, for instance to a numerically feasible quantification of a notion of quasi-flatness of manifolds, with applications in Imaging; and to the introduction of curvatures, in particular the Lipschitz-Killing curvature measures, for almost Riemannian manifolds, with a view to their usage in Regge Calculus, as well as in Graphics. Further directions of study are also suggested.
\end{abstract}

\section{Introduction}

This paper represent the first of a series of two articles dedicated (as their very title indicates) to the exploration of Metric Geometry and some of its diverse applications, mainly (but not only) in Imaging, Graphics and all the spectrum of related fields lying, so to say, in between those two.

To this end, we first have to make clear what we mean by "Metric Geometry". Understanding this concept essentially reduces to answering the following two basic questions: "What Geometry?" and "Why metric?". We begin by answering the first of these questions

1.0.0.1. What geometry? A brief answer to this first question would be "Metric Differential Geometry", but of course this further specification only invites the questions "Why Differential Geometry?" and, yet again, "Why metric?"... We answer by giving a brief answer to the second question:

${ }^{*}$ Research partially supported by Israel Science Foundation Grants 221/07 and $93 / 11$. 
1.0.0.2. Why metric? The main reason of choosing the metric approach is that it represents, so to say, a minimalistic perspective, where no supplementary smoothness or structure is imposed upon a given geometric object. We believe, therefore, that this makes it an approach ideally suited for the study of the various structures and problems encountered in computer Science in general, and in Graphics, Imaging and Vision in particular and that, moreover, this "Hypotheses non fingo" ${ }^{1}$ attitude is not only philosophically correct, it also is ideally suited for the intelligence of Digital Spaces. We shall try and augment this brief, vague argument with examples drawn from Wavelet Theory (and practice), DNA Microarray Analysis and Imaging.

We can now turn, in some more detail, to the first question:

1.0.0.3. Why differential geometry? To answer this question, one must infer first that there is another kind of Metric Geometry. Indeed, there exists a quite extensive (and, we might add, most impressive) literature on Metric Geometry and its connections to Computer Science, see [19], [62], [60], [1] to mention just a very few samples out of an extremely extensive literature. However, we do not feel satisfied with this "linear", or "first order Geometry". To explain this, we could emphasize the essential importance of "second order Geometry", or succinctly, the Geometry of curvature in Graphics and Imaging. This inevitably invites the next question:

1.0.0.4. Why curvature? We could explain in laborious detail why curvature is extremely important for theoretical ends, as well as for applications in Manifold Learning. However, we prefer not doing either of these things here, but rather referring the reader to [97] for the first issue, and to [90] and [89] for the second.

Instead, we believe (precisely as we did when writing the introduction to [83]) that the best answer is that given by R. Brooks [23]: "The fundamental notion of differential geometry is the concept of curvature", and that, in truth, it can be stated (only slightly exaggerating) that the subject of Differential Geometry is the study of curvature.

In fact, one can go even further and ask the more fundamental question

1.0.0.5. "Why geometry?" We shall not even begin to explore the importance of Geometry and geometrical thinking in Graphics, Imaging, etc., since we sincerely hope that the potential reader is fully aware and convinced of this, and since, moreover, the sequel of this paper is filled with instances galore of geometric reasoning and its applications.

\footnotetext{
${ }^{1}$ I. Newton, "Philosophiae Naturalis Principia Mathematica", 1687.
} 
In the end, as so many other things, this reduces to a matter of taste, or esthetics, so to say. ${ }^{2}$ Therefore, we should make clear that we find Geometry beautiful and enticing, not only because it represents the language in which the visual, perceivable Universe "speaks" to us, but also - and this may perhaps appear as a seemingly paradoxical motivation - it is inherently difficult: Indeed, as V. I. Arnold pointed out so poignantly, "Our brain has two halves: one is responsible for the multiplication of polynomials and languages, and the other half is responsible for orientation of figures in space and all the things important in real life. Mathematics is geometry when you have to use both halves." [63]. It is precisely this inherent difficulty of combining spatial intuition with the algebraic (ultimately symbolic or lexical) manipulation of sometimes long and convoluted formulas that makes geometry both hard for most of us and (therefore?!..) more intriguing and interesting. This is precisely the reason why "while all of us have an intuitive concept of the difference between straight and curved, it is surprisingly difficult to make the intuition precise" ([23]).

A few words regarding the organization of the material: The first section is dedicated, befittingly, to the most basic notions and results regarding the Geometry of metric spaces, in principal the Gromov-Hausdorff convergence, since it plays an essential, even if at times background role in the sequel. Section 2 is dedicated to the introducing of our "main character", namely curvature. This is done hierarchically, first for curves, then for surfaces, followed by various types of curvatures for higher dimensional manifolds. In contrast to what is perhaps more common (and too our one approach in [83]), as far as dimensions 1 and 2 are concerned, instead of concentrating all the theoretical material in a separate section, we chose to present, immediately after the classical definition of each type of curvature, its analogue or rather, as we shall see immediately - its analogues, followed immediately by some direct, immediate applications (even if some will be only sketched here). For dimensions higher or equal 3, we have chosen the more traditional way of concentrating the whole theoretical material together with that covering the lower dimensions and reserve the introduction of their metrical counterparts for the remainder of the sections. The reason for doing this is the fact that these higher dimensional metric analogues are far from classical and are, in fact, quite new, some being presented here, at least in a unified fashion, for the first time. This is done in the last section. We have chosen the apparently counterintuitive path of first discussing the metric

\footnotetext{
${ }^{2}$ As everyone is aware of the fact that, for instance, mathematicians refer to a proof as being "beautiful".
} 
version of the less well-known Lipschitz-Killing curvatures, and only later, in the second paper, on returning to the metric Ricci curvature and flow (as well on some more recent developments regarding further connections between various types of metric and related curvatures). The reason for this choice resides in the fact that it turns out it is far more easier to perform the first task, that follows almost immediately from the consideration of an important class of triangulations that we also discuss in detail, namely the so called thick triangulations.

Before we proceed to the main part of the exposition, a few cautionary and clarifying words are in order: The reader might be aware of (or shortly become so) of the previous book-chapter on curvature (including metric one) [83] that I wrote some time back. However, I would like to make clear that the present endeavor is not, in any way, a copy or even a simple extension of that work. While, to be sure, in certain parts similarities exist (after all, the subject matter is partially common and, moreover, basic Differential Geometry has remained, essentially the same!...) there are many new directions and results (some of them quite recent [93], [48], [6]), that do not appear in the previous work in question. Neither does the material here reduce to a "cut-and-paste" operation of the more applications-oriented article [97], nor even of the much earlier paper [80] that represents, in a way, the forerunner off all the subsequent developments just mentioned. ${ }^{3}$ In fact, some of the material here, particularly the one in Section 4, is new, at least in the different view of classical fields and the connections between them, that are pointed out here for the first time (at least as far as we are aware of).

However, we may say that the present exposition represents the fruit (not fully ripen yet, to be sure!...) of a natural and continuous growth processes fuelled by our steady and ever developing interest in the multifaceted and malleable metric curvatures and their various applications.

Moreover, we must caution the reader that he might find the exposition uneven in style, pace and content: The tone of the presentation may appear at times loquacious and at other times quite terse. This represents, at least in part, a natural consequence of the format, goals and space restrictions: On the one hand, this is intended to be an introductory overview, thence the perhaps overly didactic tone, mainly in the second and third sections. On the other hand, as already mentioned, new material is presented, meaning not only that clarity has to cede its place for the benefit of compactness

\footnotetext{
${ }^{3}$ Actually, [80] represents the place in literature where the use in Graphics and Imaging of the metric approach (including the Gromov-Hausdorff convergence and related notions and results) is advocated for the first time (at least to the best of our knowledge).
} 
of the presentation, but also that some (quite advanced at times) notions can be only mentioned or, at best, be sketchily introduced. While all effort was made to make the text as introductory and self contained as possible, I am quite aware that, unfortunately, this goal is far from being attained in a number of places... Still, whenever possible, I opted for the more pedagogical discourse, in an attempt to "bridge the gap between the two halves". To these words of apology it is only natural to recommend some basic (text-)books to which the reader is referred too in case any background or complementary is needed: [13] for a encyclopedic source in (quite advanced at times) Differential Geometry, [24] for Metric Geometry, as well as [65] and [56] for Differential and $P L$ Topology, respectively.

\section{The Gromov-Hausdorff distance}

We bring below only a few rudiments of Metric Geometry, not only because we wish to restrict ourselves to those aspects that are necessary to us in the sequel and to conform to the space restrictions, but also because we can hardly compete, in any sense, with the excellent, authoritative presentation in [24], to say nothing about the original [43]. (Needless to say, both these texts are warmly recommended to the reader who wishes to expand and deepening his knowledge regarding the subjects touched in this section.)

We begin by introducing the classical Hausdorff distance (metric):

Definition 2.1. Let $(X, d)$ be a metric space and let $A, B \subseteq X$. We define the Hausdorff distance between $A$ and $B$ as:

$$
d_{H}(A, B)=\inf \left\{r>0 \mid A \subset U_{r}(B), B \subset U_{r}(A)\right\},
$$

where $U_{r}(A)$ is the $r$-neighborhood of $\mathrm{A}, U_{r}(A)=\bigcup_{a \in A} B_{r}(a)$.

Another equivalent way of defining the Hausdorff distance is as follows:

$$
d_{H}(A, B)=\max \left\{\sup _{a \in A} d(a, B), \sup _{b \in B} d(b, A)\right\} .
$$

In general $d_{H}$ is only a semi-metric ${ }^{4}$ however it becomes a proper metric if we restrict ourselves to an important class of subsets. More precisely, we have the following result

Proposition 2.2. Let $(X, d)$ be a metric space. Then $d_{H}$ is a metric on the set $\mathcal{M}(X)$ of closed subsets of $X$.

\footnotetext{
${ }^{4}$ i.e. $d_{H}(X, Y)$ does not necessarily imply that $X=Y$.
} 
Moreover, the space $\left(\mathcal{M}(X), d_{H}\right)$ inherits some important properties of $(X, d)$ :

\section{Theorem 2.3.}

(a) If $X$ is complete, then $\mathcal{M}(X)$ complete.

(b) (Blaschke) If $X$ is compact, then $\mathcal{M}(X)$ compact.

(For proofs, see [24], pp. 253-254.)

It is only natural to extend the Hausdorff metric to non-compact spaces. For this, we proceed along the following basic guide-lines: We want to obtain the maximum distance $d_{G H}$ that satisfies the two conditions below:

1. $d_{G H}(A, B) \leq d_{H}(A, B)$, for any $A, B \subset X$ (i.e. sets that are close as subsets of a given metric space $X$ will still be close as abstract metric spaces);

2. $X$ is isometric to $Y$ iff $d_{G H}(X, Y)=0$.

The sought definition is then the following one:

Definition 2.4. Let $X, Y$ be metric spaces. Then the Gromov-Hausdorff distance between $X$ and $Y$ is defined by:

$$
d_{G H}(X, Y)=\inf d_{H}^{Z}(f(X), g(Y))
$$

where the infimum is taken over all metric spaces $Z$ in which both $X$ and $Y$ can be isometrically embedded and over all such isometric embeddings.

Remark 2.5. In fact, it suffices to consider embeddings $f$ into the disjoint union $X \coprod Y$ of the spaces $X$ and $Y . X \coprod Y$ is made into a metric space by defining

$$
d(x, y)= \begin{cases}i n f_{z \in X \cap Y}\left\{d_{X}(x, z)+d_{Y}(z, y)\right\}, & (x \in X) \text { and }(y \in Y) \\ \infty, & X \cap Y=\emptyset .\end{cases}
$$

The following notion is not only important for theoretical ends (see, e.g. [43]), it is also highly relevant to our applicative purposes (in Graphics, Vision, etc.)

Definition 2.6. Let $(X, d)$ be a metric space, and let $A \subset X$. $A$ is called an $\varepsilon$-net iff $d(x, A) \leq \varepsilon$, for all $x \in X$.

It should be evident, from the definition and the preparatory words preceding it, that the approximation of spaces by $\varepsilon$-nets will represent one of the main topics in the sequel. We begin by citing the following fundamental result: 
Theorem 2.7. $d_{G H}$ is a finite metric on the set of isometry classes of compact metric spaces.

Remark 2.8. It is most alluring (see [21], [22]) to make appeal to the GromovHausdorff distance between subsets of $\mathbb{R}^{3}$ (usually triangular meshes), for Graphics and Imaging goals (such as shape comparison, registration, etc.). However, this is a fallacy, since the Gromov-Hausdorff distance between two sets is not achieved by their embeddings in any Euclidean space. This was pointed out by Gromov, in [43], immediately after introducing his modification of the classical Hausdorff metric, via a simple example (see Remark (c), p. 72): Let $X$ be an equilateral triangle of edge 1, and let $Y$ be a point. Then $d_{G H}(X, Y)=1 / 2$, although the Hausfdorff distance between their embeddings in $\mathbb{R}^{2}$, hence between their embeddings in any $\mathbb{R}^{n}$, must satisfy the inequality $\left.d_{G H}^{\mathbb{R}^{n}} X, Y\right)>\sqrt{3} / 3$.

Since, moreover, computational issues also arise when trying to compute the Gromov-Hausdorff distances, between, say, two large triangular meshes, one is forced, in practice, to compute the computation of the simple Hausdorff distance, which it is not, itself, without certain complications (see e.g [32] and the bibliography therein).

In addition, $\varepsilon$-nets in compact metric spaces have the following important property:

Proposition 2.9. Let $X,\left\{X_{n}\right\}_{n=1}^{\infty}$ be compact metric spaces. Then $X_{n} \overrightarrow{G H} X$ iff for all $\varepsilon>0$, there exist finite $\varepsilon$-nets $S \subset X$ and $S_{n} \subset X_{n}$, such that $S_{n} \underset{G H}{\longrightarrow} S$ and, moreover, $\left|S_{n}\right|=|S|$, for large enough $n$.

The importance of the proposition above does not reside solely in the fact that compact metric spaces can be approximated by finite $\varepsilon$-nets (after all, just the existence of some approximation by such sets is hardly surprising), but rather in the fact that, as we shall shortly see, it assures the convergence of geometric properties of $S_{n}$ to those of $S$, as $X_{n} \underset{G H}{\overrightarrow{G H}} X$. A very important and extremely significant for us here - consequence is that of the intrinsic metric i.e. the metric induced by a length structure (i.e. path length) by a metric on a subset of a given metric space. (The motivating example, both in the theoretical setting and for this study is that of surfaces in $\mathbb{R}^{3}$.)

Moreover, the sequence $\left\{S_{n}\right\}_{n>1}$ of $\varepsilon$-nets corresponds to the situation encountered frequently in practice, where in many instances one has to approximate a smooth object (manifold), having a finite number of fixed ("sampling") point, by a sequence of $P L$ (metric, discrete) approximations, having also a fixed set of "marked" points that also converge to the "samples" chosen on the target manifold. 
Proposition 2.9 can also be reformulated in an equivalent, but less concise and elegant manner that is, on the other hand, far more useful in concrete instances as well as being more familiar for the Applied Mathematics community:

Proposition 2.10. Let $X, Y$ be compact metric spaces. Then:

(a) If $Y$ is a $(\varepsilon, \delta)$-approximation of $X$, then $d_{G H}(X, Y)<2 \varepsilon+\delta$.

(b) If $d_{G H}(X, Y)<\varepsilon$, then $Y$ is a $5 \varepsilon$-approximation of $X$.

Recall that $\varepsilon-\delta$-approximations are defined as follows:

Definition 2.11. Let $X, Y$ be compact metric spaces, and let $\varepsilon, \delta>0$. $X, Y$ are called $\varepsilon-\delta$-approximations (of each-other) iff: there exist sequences $\left\{x_{i}\right\}_{i=1}^{N} \subset X$ and $\left\{y_{i}\right\}_{i=1}^{N} \subset Y$ such that

(a) $\left\{x_{i}\right\}_{i=1}^{N}$ is an $\varepsilon$-net in $X$ and $\left\{y_{i}\right\}_{i=1}^{N}$ is an $\varepsilon$-net in $Y$;

(b) $\left.\mid d_{X}\left(x_{i}, x_{j}\right)-d_{(} y_{i}, y_{j}\right) \mid<\delta$ for all $i, j \in\{1, \ldots, N\}$.

An $(\varepsilon, \varepsilon)$-approximation is called, for short an $\varepsilon$-approximation.

Amongst metric spaces, those whose metric $d$ is intrinsic, are called length spaces and are of special interest in Geometry. The following theorem shows that length spaces are closed in the Gromov-Hausdorff topology:

Theorem 2.12. Let $\left\{X_{n}\right\}_{n=1}^{\infty}$ be length spaces and let $X$ be a complete metric space such that $X_{n} \underset{G H}{\longrightarrow} X$. Then $X$ is a length space.

Using the language of $\varepsilon$-approximations one can prove the following theorem and corollary, that are of paramount importance, not only for the goals of this overview, but in a far more extensive and powerful context (see e.g. [43] and [24]):

Theorem 2.13 (Gromov). Any compact length space is the GH-limit of a sequence of finite graphs.

The proof of the theorem above is constructive and thus potentially adaptable for practical applications (especially in Graphics, Imaging and related fields). Because of this reason we bring it below:

Proof. Let $\varepsilon, \delta(\delta \ll \varepsilon)$ small enough, and let $S$ be a $\delta$-net in $X$. Also, let $G=(V, E)$ be the graph with $V=S$ and $E=\{(x, y) \mid d(x, y)<\varepsilon\}$. We shall prove that $G$ is an $\varepsilon$-approximation of $X$, for $\delta$ small enough (more precisely, for $\left.\delta<\frac{\varepsilon^{2}}{4 \operatorname{diam}(X)}\right)$. 
But, since $S$ is an $\varepsilon$-net both in $X$ and in $G$, and since $d_{G}(x, y) \geq$ $d_{X}(x, y)$, it is sufficient to prove that:

$$
d_{G}(x, y) \leq d_{X}(x, y)+\varepsilon .
$$

Let $\gamma$ be the shortest path between $x$ and $y$, and let $x_{1}, \ldots, x_{n} \in \gamma$, such that $n \leq \operatorname{length}(\gamma) / \varepsilon$ (and $d_{X}\left(x_{i}, x_{i+1}\right) \leq \varepsilon / 2$ ). Since for any $x_{i}$ there exists $y_{i} \in S$, such that $d_{X}\left(x_{i}, y_{i}\right) \leq \delta$, it follows that $d_{X}\left(y_{i}, y_{i+1}\right) \leq d_{X}\left(x_{i}, x_{i+1}\right)+$ $2 \delta<\varepsilon$.

Therefore, (for $\delta<\varepsilon / 4$ ), there exists an edge $e \in G, e=y_{i} y_{i+1}$. From this we get the following upper bound for $d_{G}(x, y)$ :

$$
d_{G}(x, y) \leq \sum_{n=0}^{n} d_{X}\left(y_{i}, y_{i+1}\right) \leq \sum_{n=0}^{n} d_{X}\left(x_{i}, x_{i+1}\right)+2 \delta n
$$

But $n<2$ length $(\gamma) / \varepsilon \leq 2 \operatorname{diam}(X) / \varepsilon$. Moreover: $\delta<\varepsilon^{2} / 4 \operatorname{diam}(X)$. It follows that:

$$
d_{G}(x, y) \leq d_{X}(x, y)+\delta \frac{4 \operatorname{diam}(X)}{\varepsilon}<d_{X}(x, y)+\varepsilon .
$$

Thus, for any $\varepsilon>0$, there exists a graph an $\varepsilon$-approximation of $X$ by a graph $G, G=G_{\varepsilon}$. Hence $G_{\varepsilon} \vec{\varepsilon} X$.

In fact, one can infer the more stronger (and useful in applications)

Corollary 2.14. Let $X$ be a compact length space. Then $X$ is the GromovHausdorff limit of a sequence $\left\{G_{n}\right\}_{n \geq 1}$ of finite graphs, isometrically embedded in $X$.

Remark 2.15. Some care still should be paid when using the theorem above. Indeed:

1. If $G_{n} \vec{\varepsilon} X, G_{n}=\left(V_{n}, E_{n}\right)$. If there exists $N_{0} \in \mathbb{N}$ such that

$$
(*) \quad\left|E_{n}\right| \leq N_{0}, \text { for all } n \in \mathbb{N},
$$

then $X$ is a finite graph.

2. If condition $(*)$ is replaced by:

$$
(* *) \quad\left|V_{n}\right| \leq N_{0}, \text { for all } n \in \mathbb{N},
$$

then $X$ will still be always a graph, but not necessarily finite. 
Remark 2.16. In fact, one can strengthen the result of Theorem 2.13. Indeed, it was shown by Cassorla [26] that compact inner metric spaces can be, in fact, Gromov-Hausdorff approximated by smooth surfaces that, moreover, are embedded in $\mathbb{R}^{3}$. The significance for Graphics and Imaging of this result is evident and it is not lost on Cassorla, either: It shows that one can, in fact, visualize in $\mathbb{R}^{3}$ (up to some predetermined but arbitrarily small error) any compact inner metric space. This is a truly surprising fact. Unfortunately, it usually happens with "gifts", it comes at a price: The genus of the approximating surfaces can not remain bounded. (So, in effect, to obtain a good approximation even of a simple space, via the method given in the theorem's proof, one has to increase the topological complexity of the approximating surface.)

Note that there is no geometric (curvature) restriction on the approximating surfaces. In fact, the author also states - in what represents a seemingly still unpublish result - that one can approximate the given spaces with a series of smooth surfaces having Gaussian curvature bounded from above by -1 . However, this cames at the price of loosing the embeddability in $\mathbb{R}^{3}$ of the approximating surfaces. We shall address the problem of GromovHausdorff approximating, under curvature control, of surfaces by surfaces in the next paper of the series, by presenting a result of Brehm and Kühnel, as well as our own extension [93] of their result to the metric curvatures (see the following section) context.

Before concluding this remark, we should add a few words regarding Cassorla's proof, especially so since, as we mentioned above, it may prove to be useful for visualization purposes: He begins by constructing an approximation by graphs, following Gromov, then he considers the (smooth) boundaries of canonical tube neighborhoods (or, in other words, he builds the smooth surfaces having as axes (or nerve) the graph constructed previously). Since we shall encounter a very similar construction, but rather more precise, in the proof of Brehm and Kühnel theorem, we do not elaborate here anymore on Cassorla's one.

Remark 2.17. For more (and far-reaching) results regarding both the Gromov-Hausdorff approximations of metric spaces graphs, as well as that by surfaces and other "nicer" spaces, see [43], 3.32.-3.34. $\frac{1}{2}$.

2.0.0.6. The Lipschitz distance We bring briefly here few facts about another type of distance between metric spaces, that is closely related to the Hausdorff distance but may prove more easy to compute in many situations, namely the Lipschitz distance. (We do not elaborate more on this theme because we do make appeal to it in the sequel. For further details see [43], [24] or, for a "digest" somewhat more detailed than this one, [80].) 
This definition of the Lipschitz distance is based upon a very simple (that is to say: very intuitive, motivated by routine, every day physical measurements) idea: It measures the relative difference between metrics, more precisely it evaluates their ratio. That is to say that the metric spaces $\left(X, d_{X}\right),\left(Y, d_{Y}\right)$ are close (to each other) iff there exists a homeomorphism $f: X \stackrel{\sim}{\rightarrow} Y$, such that $\frac{d_{Y}(f(x), f(y))}{d_{X}(x, y)} \approx 1$, for all $x, y \in X$.

Technically, we give the following

Definition 2.18. Let $\left(X, d_{X}\right),\left(Y, d_{Y}\right)$ be metric spaces. Then the Lipschitz distance between $\left(X, d_{X}\right)$ and $\left(Y, d_{Y}\right)$ is defined as:

$$
d_{L}(X, Y)=\inf _{\substack{f, X \mathcal{\sim} \\ f b i-\bar{L} i p .}} \log \max \left(\operatorname{dil} f, \operatorname{dil} f^{-1}\right)
$$

where the dilatation $\operatorname{dil} f$ of $f$ is defined as follows:

Definition 2.19. Let $\left(X, d_{X}\right),\left(Y, d_{Y}\right)$ be metric spaces. Given a Lipschitz map $f: X \rightarrow Y$, the dilatation dilf of $f$ is defined as

$$
\operatorname{dil} f=\sup _{x \neq y \in X} \frac{d_{Y}(f(x), f(y))}{d_{X}(x, y)} .
$$

We have the following important result (analogous to Proposition 2.2):

Proposition 2.20. The Lipschitz distance $d_{L}$ is a proper distance on the space of isometry classes of compact metric spaces.

(This means, in particular, that $d_{L}(X, Y)=0$ iff $X$ and are are isometric (not only homeomorphic) - a fact of clear importance in any applicative setting.)

Remark 2.21. The following facts are very important in understanding the Lipschitz distance and its relationship with the Gromov-Hausdorff metric:

1. Notice that in the definition of $d_{L}(X, Y)$ one presumes the existence of homeomorphisms between $X$ and $Y$, that are supposed, moreover, to be bi-Lipschitz. In the absence of such homeomorphisms one defines the Lipschitz distance between $X$ and $Y$ as $d_{L}(X, Y)=\infty$. It follows that the Lipschitz distance is not suited for measuring the distance between spaces that are not homeomorphic (a fact that one should keep in mind in applications).

2. The Lipschitz topology is stronger than the Gromov-Hausdorff one, therefore convergence in $d_{L}$ implies the convergence in $d_{G H}$ (but, in general, not the other way around). 
3. Since the computation of the Gromov-Hausdorff distance between two given metric spaces necessitates the construction of a new, "universal" one, the actual computation can be quite problematic even in simple cases. A way of circumventing this obstruction is by making appeal, as in the definition of the Lipschitz metric, to a (properly defined) notion of distortion. However, in this case, instead of unctions one makes appeal to the more general notion of correspondence-for further details see, again, [43], [24] and, for a very brief summary, [80].

4. Both of the metrics introduced above have their respective drawbacks: The Lipschitz metric presumes that the spaces are homeomorphic (since otherwise their distance infinite, hence unable to encode any further information); while the Gromov-Hausdorff distance is not, in general, finite if the spaces are not bounded (which is also quite restrictive, not just for geometric ends, but in Imaging also). Therefore, one is induced to combine the two metrics into a single one, such that each of the basic metrics "blurs" the defects of the other one. This is done in a rather standard manner ${ }^{5}$ as follows:

Definition 2.22. The Gromov-Hausdorff-Lipschitz distance between two metric spaces $X$ and $Y$ is defined as:

$$
d_{G H L}(X, Y)=d_{G H}\left(X, X_{1}\right)+d_{L}\left(X_{1}, Y_{1}\right)+d_{G H}\left(Y_{1}, Y\right)
$$

where $X_{1}, Y_{1}$ are arbitrary given metric spaces.

For further details and many beautiful applications of this metric the reader is advised to consult [43], 3.C., and we contend ourselves with concluding this "detour" with the following (suggestive, as far as all kinds applications are concerned) definition:

Definition 2.23. Two metric spaces $X, Y$ are said to be quasi-isometric iff $d_{G H L}(X, Y)<\infty$.

Remark 2.24. As we mentioned above, in many instances it is more easy to measure the Lischitz distance, rather than the Hausdorff (hence also than the Gromov-Hausdorff) one. It is only natural, therefore, that people looked into its applications into Graphics and Imaging - see [20]. Again, we suggested this previously in [80].

\footnotetext{
${ }^{5}$ For other applications of this method in the construction of new metrics from old ones, see [116].
} 


\section{Curvatures - a primary}

We begin our overview of curvature, both in its classical (smooth) and metric avatars, with the basic - and motivating (one might say "inspirational") 1dimensional case:

\subsection{Curves}

We start with the basic

3.1.1. The classical (smooth) case The definition of curvature for plane curves is based upon the classical (indeed, the idea goes back to Ancient Greece) notion of curvature of a circle: $K \equiv 1 / R$, where $R$ denotes the radius of the circle. More precisely, the curvature of the curve at the point $p$ is the curvature of the "best fitting" circle to $\gamma$ at $p$ (and, of course, the curvature of a straight line is zero). It can be described as the circle that separates between the two families of circles tangent at $p$ and having centers on the normal to the curve in the point $p$, those of greater radius lying on one side of the curve, and those of smaller radius on the other. Clearly, due to continuity arguments, the "best fitting" circle itself "cuts" the curve, i.e. part of the curve will be contained in the interior, and part in the exterior of the circle (except as such critical points like, for instance, the vertices of an ellipse). ${ }^{6}$ This however is a rather elusive property, in the sense that it is not readily computable. Therefore, following Newton (1665), we define the osculatory circle as the limit of circles that have 3 common points with the curve. If the curve $\gamma \subset \mathbb{R}^{2}$ is the image of the function $c: I=[0,1] \rightarrow \mathbb{R}^{2}$, then the osculatory circle at $\gamma_{0}=c\left(t_{0}\right)$ is defined as:

$$
\begin{aligned}
C\left(\gamma_{0}\right)=C_{\gamma}\left(\gamma_{0}\right) & =\lim _{\gamma_{1}, \gamma_{2} \rightarrow \gamma_{0}} C\left(\gamma_{0}, \gamma_{1}, \gamma_{2}\right) \\
& =\lim _{t_{1}, t_{2} \rightarrow t_{0}} C\left(t_{0}, t_{1}, t_{2}\right) ; \gamma_{i}=\gamma\left(t_{i}\right), i=1,2 .
\end{aligned}
$$

Of course, the curvature $\kappa_{\gamma}\left(\gamma_{0}\right)$ of $\gamma$ at $\gamma_{0}$ is defined to be as $1 / R\left(C\left(\gamma_{0}\right)\right)$, where $R\left(C\left(\gamma_{0}\right)\right)$ is the radius of $C\left(\gamma_{0}\right)$ (see Figure 1$)$. This - namely the fact that the smaller the radius, the "benter" is the circle - is a quite intuitive idea; just recall Saint Exupery's Little Prince on his planet. Moreover, even in (meta-) mathematics it was used by Soddy in his "Kiss Precise" on the formula for the computation the radii of 4 circles tangent to each other (see, for instance, [30], p. 15). (Also, by letting $R$ go to infinity, it also fits the convention of lines having zero curvature.)

\footnotetext{
${ }^{6}$ The author wishes to thank Slava Matveev for reminding him of this fact.
} 

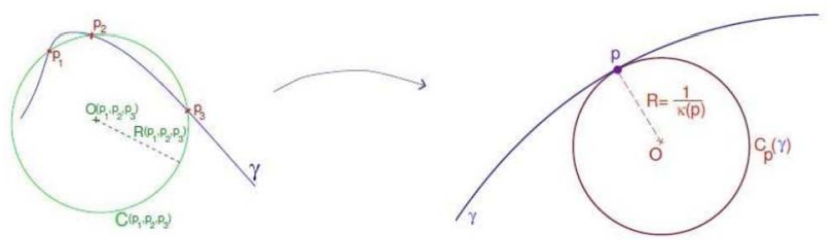

Figure 1: The osculatory circle. Note that the depicted case represents a "nice" critical point. In general the osculating circle will "cut" the curve.

It is not difficult to prove that the osculatory circle satisfies, indeed, the maximality property mentioned above (see, e.g. [111]).

Moreover, its elements, i.e. radius and center, are easily computable if the curve is given by a $\mathcal{C}^{k}$-parameterization, $k \geq 2$. More precisely, the radius of curvature $R_{C}=R\left(C\left(\gamma_{0}\right)\right)$ and the center of curvature $O_{C}=R\left(C\left(\gamma_{0}\right)\right)$ of $\gamma$ at $\gamma_{0}$ are respectively given by: $R_{C}=\frac{\left\|C^{\prime}\left(t_{0}\right)\right\|^{3}}{\left\|C^{\prime}\left(t_{0}\right) \times C^{\prime \prime}\left(t_{0}\right)\right\|}, O_{C}=\gamma_{0}+R\left(C\left(\gamma_{0}\right)\right) \mathbf{n}_{0}$, where $\mathbf{n}_{0}$ denotes the normal to $\gamma$ at the point $\gamma_{0}$. (Here " $\|\cdot\|$ " denotes the Euclidean norm on $\mathbb{R}^{2}$.)

However, it should be stressed already here that, at least in sophomoric philosophy, "curvature $i s$ the second derivative". In a weak sense this is correct, and we shall encounter the second derivative, in its many guises, further along, when discussing higher dimensional phenomena. Unfortunately, curves not always come with a specific parametrization, this being the case in various applications, such as those encountered in Graphics, Imaging, etc. where no such parametrization is to be presumed, since real-world objects do not come equipped with a "ready-made" parametrization, nor is it, in general, possible - let alone easy - to find one. This is one of the reasons - and, for us, the main reason - one is driven to consider such generalized notions of curvature, as the metric ones we concentrate in the present bookchapter. (The other reason is, of course, the purely mathematical drive of extending notions to more-and-more general spaces.)

However, one should not through out the baby together with the bathwater, as the saying goes, and in a sense, the dictum above remains correct, for a good notion of "abstract" curvature - be it metric, combinatorial, discrete, etc. - should still recapture some essential properties of the second derivative $^{7}$ (as we shall, indeed, see in the sequel).

3.1.2. Metric curvatures The simplest and most direct way of defining curvature of curves in a metric space is to closely mimic the definition of

\footnotetext{
${ }^{7}$ or, more correctly, " $a$ " second derivative - see below.
} 
the osculatory circle by first defining the (metric) curvature of triangles (or triples of points), by defining the curvature $K(T)$ of a triangle $T$ to be just $1 / R(t)$, where $R(T)$ is the radius of the circle circumscribed to the triangle. This is easily done by using some elementary high-school formulas (again, see [30]), as follows:

Definition 3.1 (Menger curvature). Let $(M, d)$ be a metric space, and let $p, q, r \in M$ be three distinct points. Then:

$$
\begin{aligned}
& K_{M}(p, q, r) \\
& \quad=\frac{\sqrt{(p q+q r+r p)(p q+q r-r p)(p q-q r+r p)(-p q+q r+r p)}}{p q \cdot q r \cdot r p}
\end{aligned}
$$

is called the Menger curvature of the points $p, q, r$. (Here and throughout this section the distance between the points $p, q$ is denoted, for brevity, by $p q$, etc.)

To define the Menger curvature at a given point on a curve, one passes to the limit (precisely like in the classical osculatory circle definition):

Definition 3.2. Let $(\mathrm{M}, \mathrm{d})$ be a metric space and let $p \in M$ be an accumulation point. We say that $M$ has at $p$ Menger curvature $\kappa_{M}(p)$ iff for any $\varepsilon>0$, there exists $\delta>0$, such that for any triple of points $p_{1}, p_{2}, p_{3}$, satisfying $d\left(p, p_{i}\right)<\delta, i=1,2,3$; the following inequality holds: $\mid K_{M}\left(p_{1}, p_{2}, p_{3}\right)-$ $\kappa_{M}(p) \mid<\varepsilon$.

One could object that, in the smooth setting, one only needs two points to converge to a third, fixed one, hence one could simplify accordingly Menger's definition of curvature as follows:

Definition 3.3 (Alt curvature). Let $(\mathrm{M}, \mathrm{d})$ be a metric space and let $p \in$ $M$ be an accumulation point. Then $M$ is said to have at the point $p$ Alt curvature $\kappa_{A}(p)$ iff the following limit exists

$$
\kappa_{A}(p)=\lim _{q, r \rightarrow p} K(p, q, r) ;
$$

where $K(p, q, r)=1 / R$ and $R$ is the circumradius of the triangle of vertices $p, q, r$.

However, it turns out that Alt's curvature is, in fact, a more general notion then Menger's curvature. Since the generality in question applies to rather esoteric spaces, and since, on the other hand, Menger's curvature proved to be strong enough to deal (quite efficiently) with such problems 
as finding estimates (obtained via the Cauchy integral) for the regularity of fractals and the flatness of sets in the plane (see [69]), it's generality seems to suffice for the (interested) mathematical community. Still, at least in the majority practical instances, the two types of curvatures coincide. The most notable - and important - instance is that of the Euclidean plane, where this equivalence (that prompted Alt's idea) is used more then once to find different interpretations of curvature (see, for instance, [111], [55], [31]).

Unfortunately, both $\kappa_{M}(p)$ and $\kappa_{A}(p)$ suffer from the same imperfection: since they are both modeled closely after the Euclidean Plane, they convey this Euclidean type of curvature upon the space they are defined on. To address this problem, another notion of metric curvature that does not closely mimic $\mathbb{R}^{2}$, therefore is better fitted for generalizations (e.g. for the metrization of graphs - see [97]), has been developed by Haantjes [51], adapting to metric spaces an idea introduced by Finsler in his PhD Thesis:

Definition 3.4 (Haantjes curvature). Let $(M, d)$ be a metric space and let $c: I=[0,1] \stackrel{\sim}{\rightarrow} M$ be a homeomorphism, and let $p, q, r \in c(I), q, r \neq p$. Denote by $\widehat{q r}$ the arc of $c(I)$ between $q$ and $r$, and by $q r$ line segment from $q$ to $r$.

Then $c$ has Haantjes (or Finsler-Haantjes) curvature $\kappa_{H}(p)$ at the point $p$ iff:

$$
\kappa_{H}^{2}(p)=24 \lim _{q, r \rightarrow p} \frac{l(\widehat{q r})-d(q, r)}{(l(\widehat{q} r))^{3}} ;
$$

where "l( $(\widehat{q r})$ " denotes the length - in the intrinsic metric induced by $d$ - of $\widehat{q r}$.

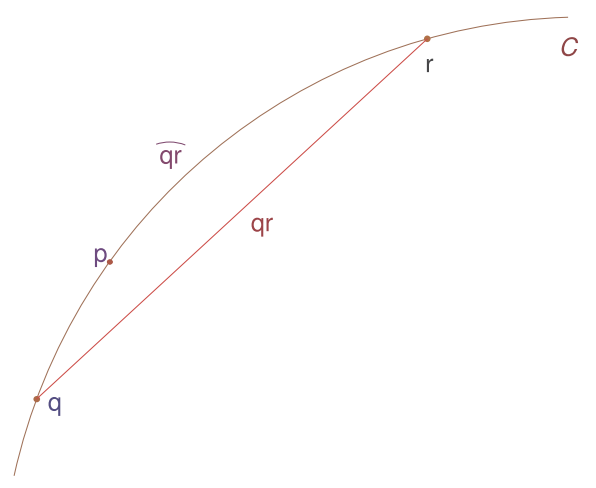

Figure 2: Haantjes curvature (of an arc). 
Remark 3.5. Alternatively, since for points/arcs where Haantjes curvature exists, $\frac{l(\widehat{q} r)}{d(q, r)} \rightarrow 1$, as $d(q, r) \rightarrow 0$ (see [51]), $\kappa_{H}$ can be defined (see, e.g. [57]) by

$$
\kappa_{H}^{2}(p)=24 \lim _{q, r \rightarrow p} \frac{l(\widehat{q r})-d(q, r)}{(d(q, r)))^{3}} ;
$$

In applications it is this alternative form of the definition of Haantjes curvature that will prove to be more malleable, as we shall illustrate shortly.

Note that, while highly intuitive and definable for a very large class of curves in general rather metric spaces, this definition of curvature would remain nothing more than a nice exercise in esoteric pastime, were it not for the following result:

Theorem 3.6. Let $\gamma \in \mathcal{C}^{3}$ be smooth curve in $\mathbb{R}^{3}$ and let $p \in \gamma$ be a regular point. Then the metric curvature $\kappa_{H}(p)$ exists and equals the classical curvature of $\gamma$ at $p$.

Simply put, for smooth curves in the Euclidean plane (or space), Haantjes curvature coincides with the standard (differential) notion, proving that, it represents, indeed, a proper generalization of the classical concept of curvature.

3.1.2.1. Curvature's comparison The question of the relationship between the two basic types of curvatures considered above, namely those proposed by Menger and Haantjes, respectively, arise naturally. Apparently, the Haantjes Curvature is a much more restricted notion than the Menger Curvature, since it applies only to rectifiable curves (contradicting to some extent the observation above regarding Menger curvature's essentially Euclidean nature). However, it turns out that the existence of $\kappa_{M}$ in a point of a ("nice enough") space implies that the space in question is, locally, a rectifiable arc, hence $\kappa_{H}$ is also definable at that point. More precisely, we have the following theorem due to Pauc [70]:

Theorem 3.7 (Pauc). Let $(X, d)$ be a metric continuum, and consider $p \in$ $X$. If $\kappa_{M}(p)$ exists, then $X$ is a rectifiable arc in a neighbourhood of $p$.

Corollary 3.8. Let $(X, d)$ be a metric arc. If $\kappa_{M}(p)$ exists at all points $p \in X$, then $X$ is rectifiable.

So, existence of one of the said curvatures assures the existence of the other (one of the implications being trivial). Moreover, it turns out that, in 
fact, the two definitions coincide, whenever then are both applicable, as the following theorem shows:

Theorem 3.9 (Haantjes). Let $\gamma$ be a rectifiable arc in a metric space $(M, d)$, and let $p \in \gamma$. If $\kappa_{M}$ and $\kappa_{H}$ exist, then they are equal.

This allows us to freely use any one of them, according to the specific requirements of a certain application. (However, as we have indicated above, we find Haantjes curvature to be far more flexible and adaptable to the modeling of various practical problems.)

3.1.2.2. Geodesics' characterization Another natural question is, whether another role of curvature in Differential Geometry, namely characterization of geodesics segments, via geodesic curvature (see, e.g. [13]) can be recaptured by metric curvatures. The answer is positive, at least for a large class of metric spaces, as the following theorem of Haantjes [52] shows:

Theorem 3.10. Let $(X, d)$ be a metric space and let $\gamma \subset X$ be a ptolemaic arc (in the induced metric). $\gamma$ is a geodesic segment if $\kappa_{H}(p)=0$, for any $p \in \gamma$.

(We do not dwell here into the definition of ptolemaic metric spaces, since this would take us too far afield, and we refer the reader to [17] or [18].)

Given Theorem 3.9, one can formulate the results above also in terms of Menger curvature. (A direct proof is also available - see, e.g. [17].)

3.1.2.3. Curvature and excess A notion of metric geometry that has been proven to be flexible and efficient, in many mathematical contexts, not least in the study of the Global Geometry of Manifolds (see, e.g., [44], [45] and the bibliography therein), is the one of excess:

Definition 3.11 (Excess). Given a triangle ${ }^{8} T=\triangle(p x q)$ in a metric space $(X, d)$, the excess of $T$ is defined as

$$
e=e(T)=d(p, x)+d(x, q)-d(p, q) .
$$

A local version of this notion was introduced (seemingly by Otsu [67]), namely the local excess (or, more precisely, the local d-excess):

$$
e_{d}(X)=\sup _{p} \sup _{x \in B(p, \rho)} \inf _{q \in S(p, \rho)}(e(\triangle(p x q)),
$$

\footnotetext{
${ }^{8}$ not necessarily geodesic
} 
where $\rho \leq \operatorname{rad}(X)=\inf _{p} \sup _{q} d(p, q)$, (and where $B(p, \rho), S(p, \rho)$ stand as they usually do - for the ball and respectively sphere of center $p$ and radius $\rho$ ).

In addition, global variations of this quantity have also been defined:

$$
e(X)=\inf _{(p, q)} \sup _{x}(e(\triangle(p x q)),
$$

and, the so called global big excess (see [67]):

$$
E(X)=\sup _{q} \inf _{p} \sup _{x}(e(\triangle(p x q)) .
$$

(Of course, in all practical settings, "sup" and "inf" replace "max" and "min" respectively, for all the instances above.)

Evidently, (local) excess and content are closely related notions, since the geometric "content", so to say, of the notion of local excess being that, for any $x \in B(p, d)$, there exists a (minimal) geodesic $\gamma$ from $p$ to $S(p, d)$ such that $\gamma$ is close to $x$. To be more precise, using a simplified notation and discarding (for convenience/simplicity) the normalizing constant "24", one has the following relation between the two notions:

$$
\kappa_{H}^{2}(T)=\frac{e}{d^{3}},
$$

where $\rho=\rho(p, q)$, and where by the curvature of a triangle $T=T(p x q)$ we mean the curvature of the path $\widehat{p x q}$. Thus Haantjes curvature can be viewed as a scaled version of excess. Keeping this in mind, one can define also a global version of this type of metric curvature, namely by defining, for instance:

$$
\kappa_{H}^{2}(T)=\frac{e}{\rho^{3}},
$$

or

$$
\kappa_{H}^{2}(X)=\frac{e(X)}{\operatorname{diam}^{3}(X)},
$$

as preferred.

Of course, one can proceed in the "opposite direction", so to speak, and express the proper (i.e. point-wise) Haantjes curvature via the definition (3.6) of local excess, as

$$
\kappa_{H}^{2}(x)=\lim _{\rho \rightarrow 0} e(x) .
$$


Remark 3.12. The metric curvatures discussed above were, unfortunately, largely forgotten for a long period of time. As already mentioned, Menger's curvature made, rather recently, quite a "revival" in Analysis. Sadly, this can not be said about the Haantjes curvature (even though, in many practical applications, it is the more adjustable and useful of the two). Still, there is a ray of light, out of this darkness, so to say: Modern (and more "tight") versions of it (and of Menger's curvature) are devised in the modern literature [4], in conjunction with the extensively used notion of Alexandrov space (see below).

We prefer the classical versions of these types of curvatures, i.e the Menger and Haantjes curvatures, to their more modern counterparts, for a number of (related) reasons:

- They are simpler and far more intuitive, thence conducive towards applications;

- They are more ready to lend themselves to discretization, hence admit easy and direct "semi-discrete" (or "semi-continuous") versions, as the one presented in Section 3.1.3 above;

- They are applicable to more general spaces, fact that represents a further incentive in their application in discrete (i.e. Computer Science driven) settings;

- While the Alexander-Bishop variants are more "tight", so to say, they coincide with their classical counterparts on all but the most esoteric spaces;

- No apriori knowledge of the global geometry of the ambient space (i.e. Alexandrov curvature) is presumed, nor is it necessary to first determine the curves of constant curvature (see [4]) in order to compute these curvatures; furthermore

- They are easy to compute in a direct fashion, at least in the discrete setting (at least amongst those discrete versions we encountered).

3.1.3. Metric curvatures - applications As already noted, Haantjes curvature lend itself naturally to a various applications and, indeed, we have applied it to a number of practical problems, which we briefly review here.

3.1.3.1. Imaging The most obvious of the applications of the metric curvatures discussed above is as approximations to principal curvatures (see Section 3.2.1), hence the computation of Gauss and mean curvature, of triangulated (polyhedral) surfaces, and their manifold uses in Imaging, Graphics and related fields. While computing them point-wise (pixel-wise) and 
without additional filtering for images gives only mediocre results (see [97], Figure 8), using larger neighborhoods (masks) improves the approximation (see Figure 3 below). Here an average Haantjes curvature is computed, defined as $\kappa_{A v g}=\left(\kappa_{\operatorname{Max}}(p i x)-\kappa_{\min }(p i x)\right) / \kappa_{\operatorname{Max}}($ pix $)$, where first curvature is computed in 4 directions, namely horizontal, vertical and two diagonal directions $\kappa_{h}, \kappa_{v}, \kappa_{d_{1}}, \kappa_{d_{2}}$. (The specific average considered here was inspired by the standard Image Processing definition of the contrast $C(I)$ of an image $\left.I, C(I)=\left(I_{\text {Max }}-I_{\min }\right) / I_{\text {Max }}.\right)$ The computation of the average Haantjes curvature for images, on a range of increasing neighbourhoods is intertwined with our next application:

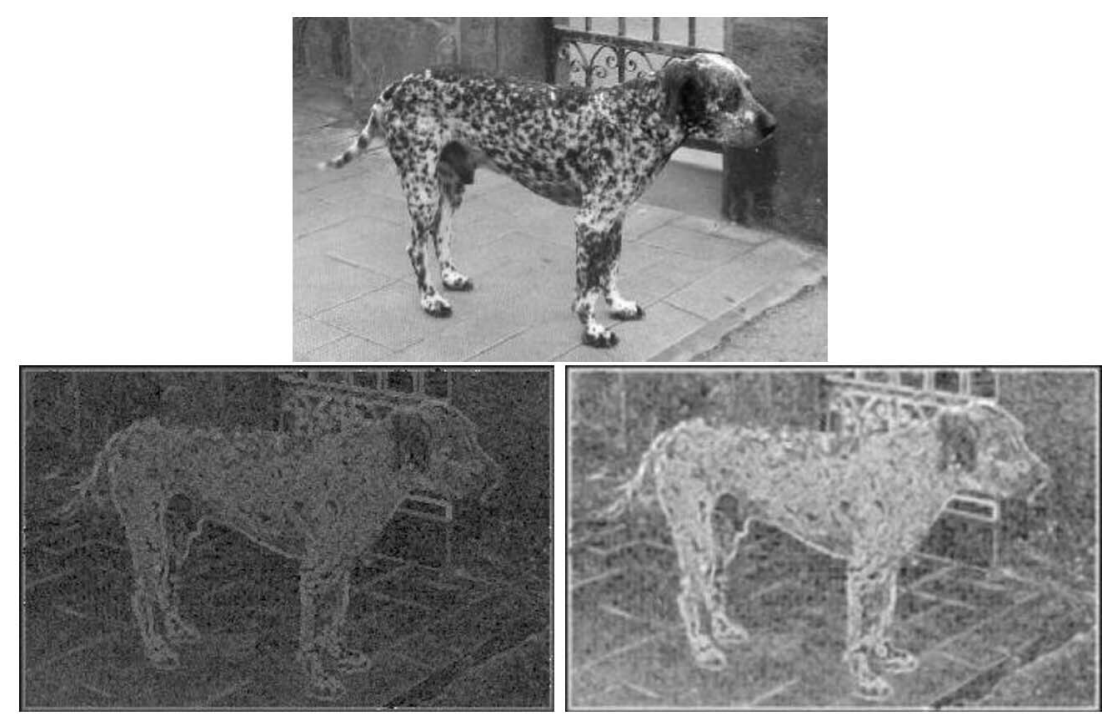

Figure 3: The average Haantjes curvature $\kappa_{A v g}$ of a natural image (above), computed locally (below, left), and on $5 \times 5$ windows (below, right).

Computing principal curvatures, mainly $\kappa_{\text {Max }}$, using either Haantjes or Menger curvature has yet another use in Imaging, more precisely in Medical Imaging, where the flattening with controlled distortion of (noisy, in many cases) images is an important task in certain applications. To this end, we have shown - using a theoretical result of Gehring and Väisälä [38] - that "patches" of triangular (polygonal) meshes can be mapped quasiconformally and, indeed, quasi-isometrically on the plane, as long as the normal $n$ to the given $P L$ surface $S$ does not deviate, on any simply connected region (patch) $U \subseteq S$, "too much" from an initial value $n_{0}$ (see [98]). Note 
that quasi-conformal mappings, are, indeed, as their name implies "almost preserving" angles (for the proof in the 2-dimensional case, see [3]. Recall also that quasi-isometric mappings are defined as follows:

Definition 3.13. Let $D \subset \mathbb{R}^{n}$ be a domain. A homeomorphism $f: D \rightarrow \mathbb{R}^{n}$ is called a quasi-isometry (or a bi-lipschitz mapping), if there exists $1 \leq C<$ $\infty$ such that

$$
\frac{1}{C}\left|p_{1}-p_{2}\right| \leq\left|f\left(p_{1}\right)-f\left(p_{2}\right)\right| \leq C\left|p_{1}-p_{2}\right|, \text { for all } p_{1}, p_{2} \in D ;
$$

where "| $\cdot \mid$ " denotes the standard (Euclidean) metric on $\mathbb{R}^{n}$.

$C(f)=\min \{C \mid f$ is a quasi-isometry $\}$ is called the minimal distortion of $f$ (in $D$ ).

(In the definition above we have restricted ourselves solely to the setting relevant here.)

In our adaptation of Gehring and Väisälä's theoretical result, from smooth surfaces to triangular meshes, we had to make a choice of the normal and of a tangent plane at a vertex of the triangulation; which we did by choosing (as it is common in Graphics) $n_{0}$ to be the mean of the normals to the faces adjacent to the considered vertex. The choice of the starting point (which determines the size of the patch $U$ ) was shown to be best done by considering the Gaussian curvature, more precisely its (classical by now, see e.g. [55], [49]) discretization as angular defect. (For details, including precise algorithms and examples, see [98].)

This somewhat empiric approach can be made rigorous by making appeal to results of Semmes (see [43], Appendix B and the relevant bibliography within). Technically put, the deviation of the normal to a surface $S$ can be controlled by the $\|\cdot\|$ norm:

$$
\|\mathbf{n}\|_{*}=\sup _{x \in S, R>0} \frac{1}{|B(x, R) \cap S|} \int_{|B(x, R) \cap S|}\left|n(y)-n_{x, R}\right| d y,
$$

where $n_{x, R}$ is the normal mean on $B(x, R) \cap S$, that is

$$
n_{x, R}=\frac{1}{|B(x, R) \cap S|} \int_{B(x, R) \cap S} n(y) d y .
$$

The geometric condition we seek is expressed using $\|\mathbf{n}\|_{*}$ via

Definition 3.14. $S^{d}$ is called a chord-arc surface with small constant $(C A S S C)$ if 
(i) $\|\mathbf{n}\|_{*} \leq \varepsilon$, and

(ii)

$$
\sup _{x \in S, R>0} \sup _{y \in B(x, R) \cap S} \frac{1}{R}\left|<x-y, n_{x, R}>\right| \leq \varepsilon_{1} .
$$

Indeed, condition (3.16) of the preceding definition takes place iff for any $x \in S$ and for all $R>0$, the set $B(x, R) \cap S$ remains close to the hyperplane through $x$ normal to $n_{x, R}$. Formally, there exist a hyperplane $H=T_{n_{x, R}}$ such that, for any $y \in B(x, R) \cap S$ the following holds:

$$
d\left(y, T_{n_{x, R}}\right) \leq \varepsilon_{2} R .
$$

In metric terms we can formulate the following equivalent definition:

Definition 3.15. The surface $S$ is called $\varepsilon$-flat if

$$
l(\gamma) \leq(1+\varepsilon)|x-y|,
$$

and

$$
\left(1-\varepsilon^{\prime}\right) \operatorname{Area}_{\mathrm{Eucl}}\left(B^{2}(R)\right) \leq|B(x, R) \cap S| \leq\left(1+\varepsilon^{\prime}\right) \operatorname{Area}_{\mathrm{Eucl}}\left(B^{2}(R)\right)
$$

$R$ being as above.

Note that a surface being $\varepsilon$-flat represents a stronger statement than the simple quasi-isometry condition (3.18), due to the low area distortion expressed by condition (3.19).

Remark 3.16. Note that all the definitions and conditions above can be trivially extended to higher dimensions (only "Area" being replaced by "Vol", of course).

As expected, the deviation of the normal (hence flatness) can controlled by curvature, more precisely if $S$ has "small curvature in the mean", i.e. if

$$
\int_{S}\left(k_{M a x}(x)\right)^{2} d x=\left\|k_{M a x}\right\|_{L^{2}}<\delta,
$$

for small $\delta$, then $S$ is $\varepsilon$-flat.

Remark 3.17. As above one can extend, mutatis mutandis, this condition to any dimension.

Remark 3.18. Condition (3.20) is, in fact, stronger that $\varepsilon$-flatness, because it requires higher order derivatives. 
Remark 3.19. Condition (3.20) is scale invariant.

For a surface to have small curvature in the mean does not just imply mere $\varepsilon$-flatness; in fact condition implies (see [114]) that there exists a $(1+\eta)$ bilipschitz parameterization of $M$, where $\eta=\eta(\delta)$, and where $\eta \rightarrow 0$, when $\delta \rightarrow 0$.

It follows, therefore, that using metric curvatures (Haantjes or Menger) provides us with a powerful tool for flattening and bilipschitz parametrization of noisy and "rough" data.

Another immediate application stems from Theorem 3.10, namely using Haantjes curvature, "en lieu" of geodesic curvature, to determine geodesics on triangular (polygonal) meshes. (Some promising first experiments have already been undertaken.)

3.1.3.2. Wavelets While perhaps unexpected, the connection between scale and curvature (more specific Haantjes curvature) is quite evident, once one is willing to notice and accept it. Indeed, consider the generic $P L$ wavelet $\varphi$ in Figure 4 below, and let $\widehat{A E}$ be the arc of curve between the points $A$ and $E$, and let $d(A, E)$ is the length of the line-segment $A E$. Then

$$
l(\widehat{A E})=a+b+c+d ; d(A, E)=e+f .
$$

Using the alternative formula for the Haantjes curvature (3.4), the following discretization of the Haantjes curvature is, therefore, natural:

$$
\kappa_{H}^{2}(\varphi)=24[(a+b+c+d)-(e+f)] /(e+f)^{3} .
$$

In addition to the total curvature of the wavelet $\varphi$, one can also compute the "local" curvatures of the partial wavelets $\varphi_{1}=\widehat{A B C}$ and $\varphi_{2}=\widehat{C D E}$,

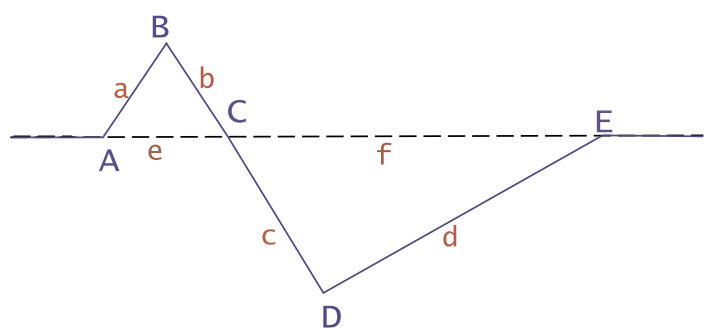

Figure 4: A generic $P L$ wavelet. 
that is the curvatures at the "peaks" $B$ and $D$ :

$$
\kappa_{H}^{2}(B)=24(a+b-e) / e^{3},
$$

and

$$
\kappa_{H}^{2}(D)=24(c+d-f) / f^{3},
$$

as well as the mean curvature of these peaks:

$$
H_{H}(\widehat{A E})=\left[\kappa_{H}(B)+\kappa_{H}(D)\right] / 2 .
$$

Even though these variations may prove to be useful in certain applications, we believe that the correct approach, in the sense that it best corresponds to the scale of the wavelet, would be to compute the total curvature of $\varphi$. Of course, there is nothing special about $P L$ wavelets (except their simplicity) and one can compute the Haantjes curvature for the Haar scaling function and wavelet, as well as for other, smooth families of wavelets (see [103], [6]). The connection scale-curvature for a number of families of curves, as well as some general estimates, were given in [103], [6]. (Note also that, via the principal curvatures of a surfaces, one can extend the scale-curvature duality to curvaturelets, ridgelets and shearlets, as well [103].)

A first application of this connection is presented in [6], where the scalecurvature connection is employed to texture analysis and segmentation in images (see, for example, Figure 5 as well as [6]). In addition, in both papers mentioned above, an automatic scale detection is suggested (as well as the obvious use of wavelet curvature as edge detector).

More important than any specific application, is the fact that the scalecurvature duality allows for a first bridging, even if only a partial one, of the gap between the two basic, largely non-intersecting, approaches prevalent in Image Processing and related fields: The geometric one, that is closely related to the Graphics community philosophy; and the more classical, Fourier Analysis/Wavelets driven one. In fact, it is quite natural to replace the vaguely defined (but intuitively clear) concept of "scale", to the classical, well defined one of "curvature", by formally defining scale by means of the Haantjes curvature, at least in the purely theoretical setting. This is more relevant in the context of 2-dimensional (as well, of course, as higher dimensional ones), nonseparable signals, where a proper notion of scale is far less intuitive then in the 1-dimensional, classical, case. 


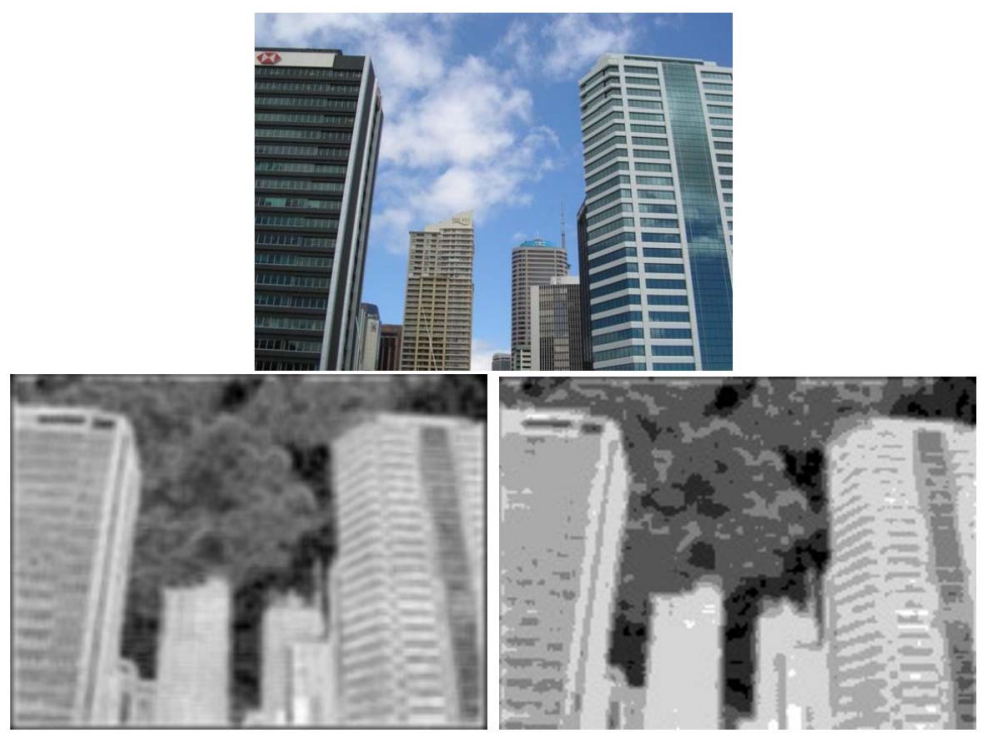

Figure 5: Texture segmentation of an urban landscape image (above): Average Haantjes curvature (bottom, left) and the texture segmentation of image (bottom, right), using 7 scales.

3.1.3.3. DNA microarray analysis Given that combinatorial curvature at a vertex $v$ of a graph $G$, i.e.

$$
C(v)=\frac{2 \# T}{\rho(v)(\rho(v)-1)},
$$

where $\# T$ denotes the number of triangles with vertex $v$ and $\rho(v)$ denotes the degree of the vertex $v$, can also be expressed in metric terms as

$$
C(v)=2-<d(v)>,
$$

where $\langle d(v)\rangle$ denotes the mean distance (in the combinatorial metric) from $v$; we have generalized, in [96], the by now common combinatorial curvature, both to vertex and edge weighted graphs, using a discretization of the Haantjes curvature, to obtain a more geometric discrete curvature for weighted graphs. Our first suggested application was for clustering, with specific applications in DNA micro-array analysis, but other uses are also natural (see the paragraph below). 
3.1.3.4. Other applications Given the proven versatility of Haantjes curvature, it is not difficult to contemplate its use in various other settings other than those mentioned above. Amongst these, we mention some obvious one, such as Pattern Recognition (via clustering, for instance), more general Manifold Learning applications (also in conjunction with other metric invariants of [44], [45]); routing (e.g. hole detection) in Communication Networks (using the metric curvature characterization of geodesics); Information Geometry; etc. (Some of these directions represent work in progress with David Gu and Jie Gao.)

3.1.3.5. Menger curvature in pattern recognition We have concentrated on the applications of Haantjes curvature, since we find it to be conceptually more flexible. However, given the equivalence between Haantjes and Menger curvatures on rectifiable metric arcs, one can apply Menger curvature as well in most of the instances mentioned above (with the exception of the application to wavelets where the discretization considered makes direct appeal to the definition of Haantjes curvature. However, we mention here an application [39] to Pattern Recognition of Menger curvature per se, or rather of the Menger curvature measure:

$$
\mu(\mathcal{T})=\mu_{p}(\mathcal{T})=\sum_{T \in \mathcal{T}} \kappa_{M}^{p}(T)(\operatorname{diam} T)^{2},
$$

for some $p \geq 1$, where $\kappa_{M}(T)$ denotes the Menger curvature (of the triangle $T$ ). (For the specific application and further details, see [39].)

\subsection{Surfaces}

Precisely as in the 1-dimensional case, we first introduce the notion of curvature in the classical (smooth) case, then we pass to its generalizations to metric space setting.

3.2.1. The classical (smooth) case We have introduced in the previous subsection the notion of osculatory circle and we have remarked that it admits further generalizations. One would expect that it would work particularly well for one of the most immediate generalization of plane curves, i.e. for surfaces in $\mathbb{R}^{3}$. Unfortunately, this idea, however nice and natural, does not work for surfaces - the osculatory sphere (introduced first by Fuss, in 1829) can not be used to define a notion of curvature for surfaces (see 7 (b) to intuitively understand the obstruction). 

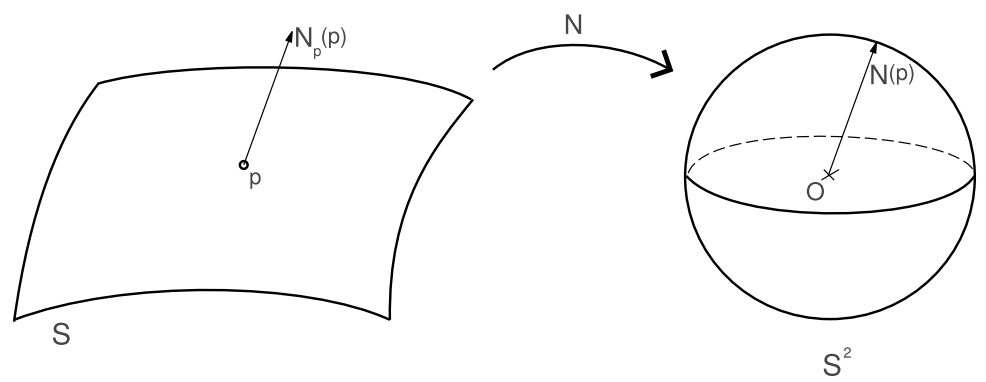

Figure 6: The Normal Mapping.

The next natural idea, which was the one in use before the seminal work of Gauss, is to "cut" the surface in all possible directions (by "dissecting" the surfaces with normal planes) and to define curvature for the surfaces $S$ via the curvatures of the resulting sections (curves). However, the idea above is far from satisfactory, at least at first view, since there exist infinitely many directions and it is hard to ascertain in what way do the sectional curvatures represent the curvature of the surface. We shall see presently how Gauss this quandary, too.

However, his approach was different and revolutionary. ${ }^{9}$ His most inspired idea was to define curvature as a measure of a surface from "being straight" or equivalently, a measure of how much a surface has to be bent in order to obtain a certain standard surface, i.e. the unit sphere $\mathbf{S}^{2}$. Gauss achieved this by considering the normal mapping $\nu: S \rightarrow \mathbf{S}^{2}$ (see Figure 6). Then the Gauss curvature of $\mathrm{S}$ at $p$ is defined as:

$$
K(p)=K_{S}(p)=\lim _{\operatorname{diam}(R) \rightarrow 0} \frac{\operatorname{Area}(\nu(R))}{\operatorname{Area}(R)}
$$

where $R$ is a simple region, $p \in R \subset S$.

A sign is attached to $K(p)$ in a natural way - see Figure 7 below.

Remark 3.20. Note that the same basic idea is also applicable for plane curves $^{10}$ - see, e.g. [111], for details and the historical development of this

\footnotetext{
${ }^{9}$ It is worthwhile pausing in order to mention that Gauss came up with the new insight after abandoning Mathematics for a while (feeling dissatisfied with it and with his achievements) and working for a while in a "high-tech" job of a period, namely in cadastral mensuration and gaining - as we shall see - new geometric insights.

${ }^{10}$ Of course, in this case, arc length (or angle measure) replaces area.
} 

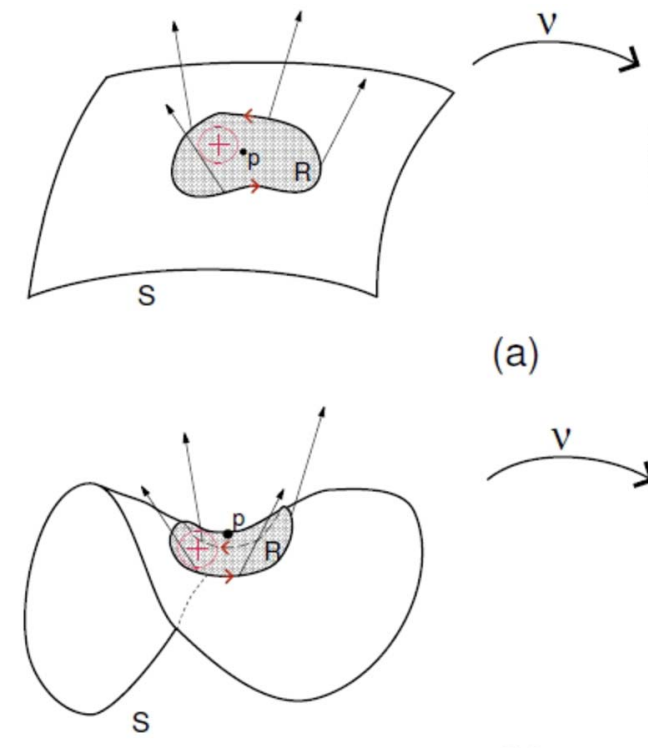

(a)

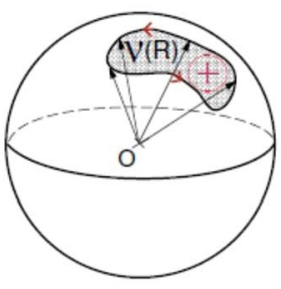

$\mathrm{S}^{2}$

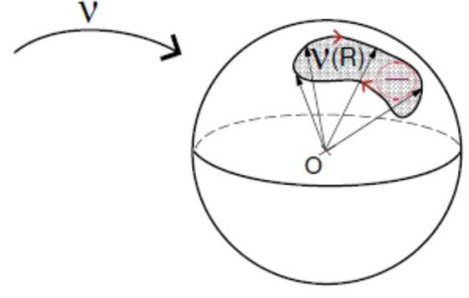

$\mathrm{S}^{2}$

(b)

Figure 7: Gauss' Curvature Definition: (a) $K(p)>0$ and (b) $K(p)<0$.

ideas and, in particular, "the state of the art" before Gauss, and his farreaching, revolutionary role in the development of Differential Geometry.

But Gauss did more than this. In particular he proved the following formula:

$$
K(p)=k_{\min } k_{\text {Max }},
$$

where $k_{\min }$ and $k_{\text {Max }}$ are the minimal, respective maximal normal curvatures of the surface $S$ at the point $p$ (see Figure 8). Recall that the normal curvature of $\gamma \subset S$ in the direction $\mathbf{v}$ at a point $p$ is defined as: $\kappa_{v}(p)=\kappa_{\gamma}(p)$, where $\gamma=S \cap P$, and where $P$ is a plane such that $P \perp T_{p}(S)$ and such that $\gamma^{\prime} \| \mathbf{v}$. Here $T_{p}(S)$ denotes the tangent plane to $S$ at $p$.

This formula is unfortunately - since it is neither immediate nor natural - employed as the definition of the Gauss curvature. (And, we might add, due to its simplicity, tends to induce in many students a lack of curiosity and motivation for deeper geometric understanding.) It should be remembered, therefore, that Gauss defined curvature via area and then proved Formula 3.30. (Again, see [111] for the history of Gauss' discovery.)

Before we proceed further, let us note that both definitions of surface curvature considered above render themselves to generalization and applica- 


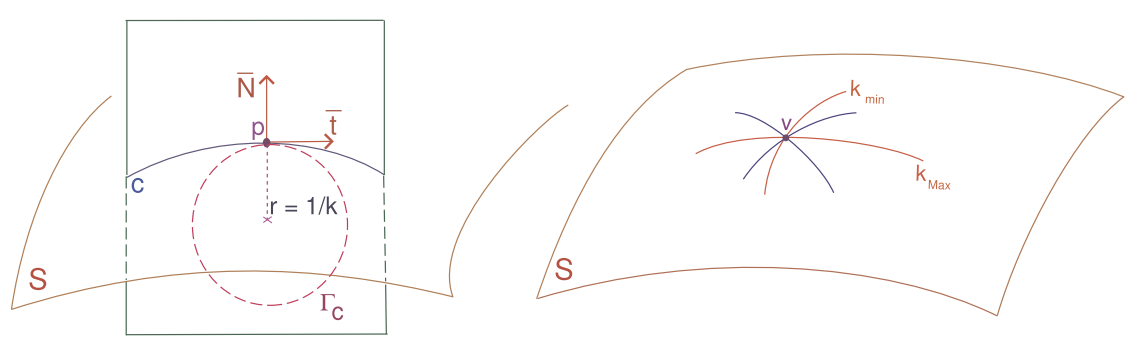

Figure 8: Principal curvatures on a smooth surface.

tion (fact which we shall exploit to our advantage in the sequel). However, the original geometric idea of Gauss is the one that will provide us in Section 3.2 .2 with an extension of the notion of curvature to rather abstract spaces (and in a manner that is also useful in applications).

The reader will recall that, in the case of curves, "curvature equals second derivative" and surely will ask herself or himself whether this is not the case also for surfaces? This is a most legitimate question, whenever a second derivative exists, i.e. in the classical case of smooth surfaces, i.e. of class $\mathcal{C}^{k}, k \geq 2$, (which certainly deserves special attention).

Let $U=\operatorname{int}(U)$ be an open set and let $f: U \subseteq \mathbb{R}^{2} \rightarrow \mathbb{R}^{3}$ be a smooth function (i.e. $f \in \mathcal{C}^{k}, k \geq 2$ ). Then the expression of $K$ in local coordinates is:

$$
K=\frac{e g-f^{2}}{E G-F^{2}}
$$

where

$$
E=f_{u} \cdot f_{u}, F=f_{u} \cdot f_{v}, G=f_{v} \cdot f_{v}
$$

and

$$
e=\frac{\operatorname{det}\left(f_{u}, f_{v}, F_{u u}\right)}{\sqrt{E G-F^{2}}}, f=\frac{\operatorname{det}\left(f_{u}, f_{v}, F_{u v}\right)}{\sqrt{E G-F^{2}}}, g=\frac{\operatorname{det}\left(f_{u}, f_{v}, F_{v v}\right)}{\sqrt{E G-F^{2}}}
$$

where $f_{u}=\partial f / \partial u$, etc. and "." denotes the scalar product.

The matrices $I_{f}=\left(\begin{array}{cc}E & F \\ F & G\end{array}\right)$ and $I I_{f}=\left(\begin{array}{cc}e & f \\ f & g\end{array}\right)$ are called the first, respectively the second fundamental form of $S$.

The reason we brought wasn't merely to make a point for the "curvature equals the second derivative" assertion. It certainly is intended to have an 
"educational" aspect, too, so to say. By this we mean that formulas such as the ones above (and like (3.34) below) should make the case for the need for a more intuitive, (geo-)metric approach to curvature. This aspect will be emphasized, inevitably, even more in the discussion regarding curvature(s) for higher dimensional manifolds. In addition to their motivational advantage, we can put to use such formulas to formulate a deep and important result of Gauss. But first, we need to bring the following (quite frightening!) formula (the so called Frobenius formula): ${ }^{11}$

$$
\begin{aligned}
K= & -\frac{1}{4\left(E G-F^{2}\right)^{2}}\left|\begin{array}{ccc}
E & E_{u} & E_{v} \\
F & F_{u} & F_{v} \\
G & G_{u} & G_{v}
\end{array}\right| \\
& -\frac{1}{\sqrt{E G-F^{2}}}\left(\frac{\partial}{\partial v} \frac{E_{v}-F_{u}}{\sqrt{E G-F^{2}}}-\frac{\partial}{\partial u} \frac{F_{v}-G_{u}}{\sqrt{E G-F^{2}}}\right) ;
\end{aligned}
$$

where $F_{u}=\partial F / \partial u$, etc.

Assuredly Frobenius formula is daunting enough to convince almost anyone that a metric approach might be preferable. However, it is more important than that, as we shall show presently. But first, we need a few more preparatory observations: Note that $I I_{f}$ depends on the position of $S$ in space (see e.g. [31], p.154), i.e. upon the specific embedding considered. Therefore, the problem with the definition of $K$ given by formula (3.31) is that it is dependent upon $I I_{f}$, hence upon the specific embedding of $S$ in $\mathbb{R}^{3}$, thus its relevance as an intrinsic geometric invariant of the surface $S$ appears to be limited, to say the least. A property is called intrinsic iff it depends solely upon the the first fundamental form of the surface, hence it is invariant under local isometries. For example, mean curvature

$$
K(p)=\frac{k_{\min }+k_{\operatorname{Max}}}{2},
$$

is not an intrinsic property (see, e.g. [13]). However, since Frobenius' formula shows $K$ is independent of $I I_{f}$, immediately implies:

Theorem 3.21 (Gauss' Theorema Egregium ("The Remarkable Theorem")). Gaussian curvature is intrinsic.

How important - and surprising - this result is, can be inferred from the name Gauss himself gave it: "Theorema Egregium" (i.e. "The Remarkable Theorem"). We shall expand on this important theorem it (or rather its

${ }^{11}$ See [17], p. 365. A variety of similar formulas exist, see e.g. proof, [111], p. 112. 
relevance and applications) in a number of occasions in the sequel. For now we only mention here that a fitting Theorema Egregium analogue has been developed for the $P L$ standard version of Gaussian curvature [55], [75], [8], [9]. Let us only add that, in more than one sense, a "proper" notion of curvature should be intrinsic hence, after introducing a new type of curvature, one should try an prove a fitting parallel to Theorema Egregium.

3.2.1.1. Another approach: the Bertrand-Diguet-Puiseaux formulas The formulas we selected give simple geometric interpretation to Gaussian curvature, that will be employed herein and on which we focus. They relate to the circumference (respective area) of a small circle on a surface (or geodesic circle - for the precise definition see, e.g. [31], p. 287).

Theorem 3.22 (Bertrand-Diguet-Puiseaux [16], 1848). Let $S$ be a surface in $\mathbb{R}^{3}, p \in S$ and let $\varepsilon>0$. Denote by $C(p, \varepsilon), B(p, \varepsilon)$ the geodesic circle, respective the geodesic ball of center $p$ and radius $\varepsilon>0$. Then:

$$
\text { length } C(p, \varepsilon)=2 \pi \varepsilon-\frac{\pi}{3} K(p) \varepsilon^{3}+o\left(\varepsilon^{3}\right),
$$

and

$$
\operatorname{area} B(p, \varepsilon)=\pi \varepsilon-\frac{\pi}{12} K(p) \varepsilon^{4}+o\left(\varepsilon^{4}\right)
$$

Hence:

$$
K(p)=\lim _{\varepsilon \rightarrow 0} \frac{3}{\pi} \frac{2 \pi \varepsilon-\text { length } C(p, \varepsilon)}{\varepsilon^{3}}=\lim _{\varepsilon \rightarrow 0} \frac{12}{\pi} \frac{\pi \varepsilon^{2}-\operatorname{area} B(p, \varepsilon)}{\varepsilon^{4}}
$$

(For a proof see e.g. [58], pp. 104-105, or [31], pp. 292 and 294.)

In their usual appearance in a standard Differential Geometry text-book, this formulas have only a limited, restricted importance. However, "a stick points in two opposite directions", therefore both these formulas can be used as new, alternative definitions of (Gauss) curvature!.. This is relevant in a more "abstract" setting, where an easily discretizable formulation would be useful (such as in Geometric Group Theory). We shall, however, make good use of the first formula as an intuitive way of understanding curvature in higher dimensions (and finding expressive, relevant definitions of curvature in more general spaces) - see below.

3.2.2. Wald curvature As we have mentioned above - and as it will become more clear in the recurring discussion on this theme, mainly in Section 4 - the efficiency of Menger and Haantjes curvature to approximating 
curvature of manifolds of dimension $\geq 2$ is rather limited, to say nothing about the their theoretical limitations (e.g. in proving relevant analogues of classical theorems, e.g. the Gauss-Bonnet Theorem).

Therefore we concentrate here, as well as in the last two sections, on another, more powerful approach. By "powerful" we mean not only that it is very flexible and thus facilitates various theoretical, as well as practical applications (see [48], [93]); nor merely that it allows us to obtain powerful theoretical results; but also that it integrates perfectly (one is almost tempted to say "ideally") with the general metric scheme detailed in Section 2, and particularly so with the Gromov-Hausdorff convergence (see [94], as well as our sequel paper [100]).

Instead of using principal curvatures (on whom limitations we already elaborated), the approach in question rather goes back to Gauss' original method of defining surface curvature by comparison to a standard, model surface (i.e. the unit sphere in $\mathbb{R}^{3}$ ). It was Wald's idea [117], [118] to use more types of gauge surfaces and to restrict oneself to the study of the minimal geometric figure that allows this comparison.

Definition 3.23. Let $(M, d)$ be a metric space, and let $Q=\left\{p_{1}, \ldots, p_{4}\right\} \subset$ $M$, together with the mutual distances: $d_{i j}=d_{j i}=d\left(p_{i}, p_{j}\right) ; 1 \leq i, j \leq 4$. The set $Q$ together with the set of distances $\left\{d_{i j}\right\}_{1 \leq i, j \leq 4}$ is called a metric quadruple.

Remark 3.24. Metric quadruples can be defined in a slightly more abstract manner, without the aid of the ambient space: a metric quadruple being a 4 point metric space, i.e. $Q=\left(\left\{p_{1}, \ldots, p_{4}\right\},\left\{d_{i j}\right\}\right)$, where the distances $d_{i j}$ verify the axioms for a metric.

We need to introduce some further notation, before being able to pass to the next definition: $S_{\kappa}$ denotes the complete, simply connected surface of constant Gauss curvature $\kappa$ (or space form), i.e. $S_{\kappa} \equiv \mathbb{R}^{2}$, if $\kappa=0$; $S_{\kappa} \equiv \mathbb{S}_{\sqrt{\kappa}}^{2}$, if $\kappa>0$; and $S_{\kappa} \equiv \mathbb{H}_{\sqrt{-\kappa}}^{2}$, if $\kappa<0$. Here $S_{\kappa} \equiv \mathbb{S}_{\sqrt{\kappa}}^{2}$ denotes the sphere of radius $R=1 / \sqrt{\kappa}$, and $S_{\kappa} \equiv \mathbb{H}_{\sqrt{-\kappa}}^{2}$ stands for the hyperbolic plane of curvature $\sqrt{-\kappa}$, as represented by the Poincaré model of the plane disk of radius $R=1 / \sqrt{-\kappa}$.

Definition 3.25. The embedding curvature $\kappa(Q)$ of the metric quadruple $Q$ is defined to be the curvature $\kappa$ of the gauge surface $S_{\kappa}$ into which $Q$ can be isometrically embedded. (See Figure 9.)

We can now define the Wald curvature [117], [118] (or, more precisely, its modification due to Berestovskii [11]): 

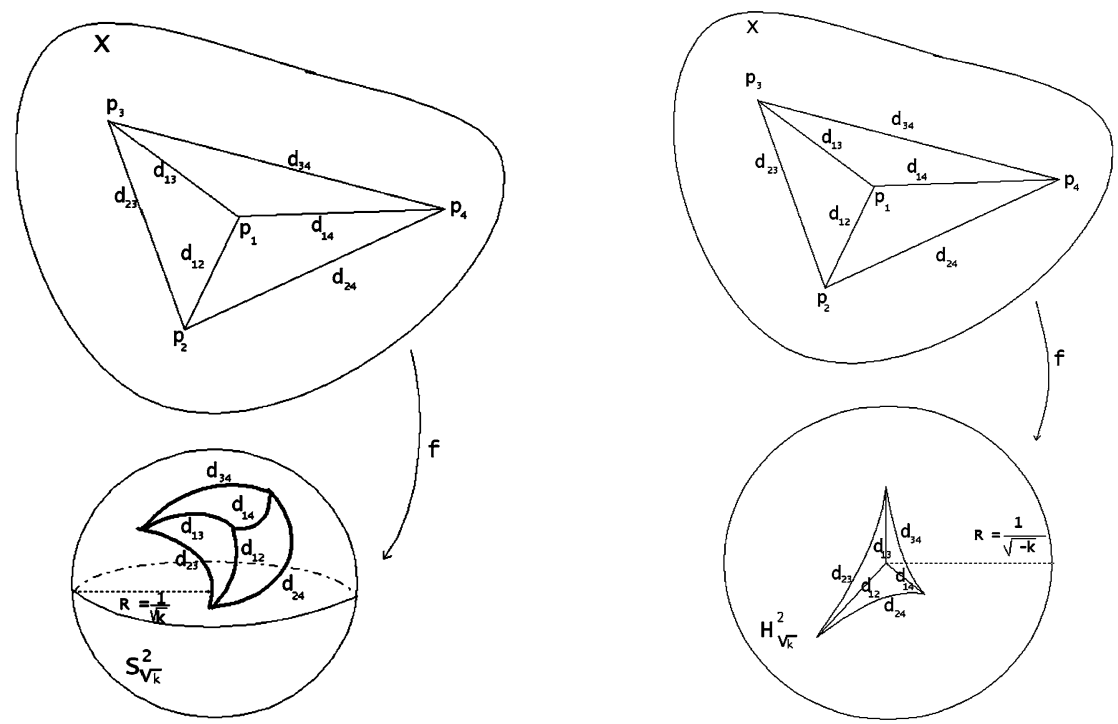

Figure 9: Isometric embedding of a metric quadruple in $\mathbb{S}_{\sqrt{\kappa}}^{2}$ (left) and $\mathbb{H}_{\sqrt{\kappa}}^{2}$ (right) .

Definition 3.26. Let $(X, d)$ be a metric space. An open set $U \subset X$ is called a region of curvature $\geq \kappa$ iff any metric quadruple can be isometrically embedded in $S_{m}$, for some $m \geq k$. A metric space $(X, d)$ is said to have Wald-Berestovskii curvature $\geq \kappa$ iff any $x \in X$ is contained in a region $U=U(x)$ of curvature $\geq \kappa$.

Remark 3.27. Clearly, in the context of polyhedral surfaces, the natural choice for the set $U$ required in Definition 3.26 is the star of a given vertex $v$, that is, the set $\left\{e_{v j}\right\}_{j}$ of edges incident to $v$. Therefore, for such surfaces, the set of metric quadruples containing the vertex $v$ is finite.

Equipped with this quite simple and intuitive choice for $U$ (and in in analogy with Alexandrov spaces - see also Section 5.2 below) it is quite natural to consider, for $P L$ surfaces, the following definition of the Wald curvature $K(v)$ at the vertex $v$ :

$$
K_{W}(v)=\min _{v_{i}, v_{j}, v_{k} \in \operatorname{Lk}(v)} K_{W}^{i j k}(v)
$$

where $K_{W}^{i j k}(v)=\kappa\left(v ; v_{i}, v_{j}, v_{k}\right)$, and where $\operatorname{Lk}(v)$ denotes the link of the vertex $v$ - see Footnote 11 below. ${ }^{12}$

\footnotetext{
${ }^{12}$ Recall that the $\operatorname{link} \operatorname{lk}(v)$ of a vertex $v$ is the set of all the faces of $\overline{\operatorname{St}}(v)$
} 
Note that here we consider the (intrinsic) $P L$ distance between vertices.

The embedding curvature at a point can now be defined naturally as a limit. However, we need first yet another preparatory definition:

Definition 3.28. $(M, d)$ be a metric space, let $p \in M$ and let $N$ be a neighbourhood of $p$. Then $N$ is called linear iff $N$ is contained in a geodesic curve.

Definition 3.29. Let $(M, d)$ be a metric space, and let $p \in M$ be an accumulation point. Then $M$ has (embedding) Wald curvature $\kappa_{W}(p)$ at the point $p$ iff

1. Every neighbourhood of $p$ is non-linear;

2. For any $\varepsilon>0$, there exists $\delta>0$ such that if $Q=\left\{p_{1}, \ldots, p_{4}\right\} \subset M$ and if $d\left(p, p_{i}\right)<\delta, i=1, \ldots, 4$; then $\left|\kappa(Q)-\kappa_{W}(p)\right|<\varepsilon$.

Remark 3.30. Even if the basic idea of embedding curvature is, in fact, quite natural, one should take care when trying to employ it directly, since there are a number of "surprises" that arise:

1. If one uses the second (abstract) definition of the metric curvature of quadruples, then the very existence of $\kappa(Q)$ is not assured, as it is shown by the following

Counterexample 3.31. The metric quadruple of lengths

$$
d_{12}=d_{13}=d_{14}=1 ; d_{23}=d_{24}=d_{34}=2
$$

admits no embedding curvature.

2. Any linear quadruple is embeddable, apart from the Euclidean plane, in all hyperbolic planes (i.e. of any strictly negative curvature), as well as in infinitely many spheres (whose radii are sufficiently large for the quadruple to be realized upon them).

3. Moreover, even if a quadruple has an embedding curvature, it still may be not unique (even if $Q$ is not linear); as it is illustrated by the following examples:

Example 3.32. (a) For each $\kappa>0$, each neighbourhood of any point $p \in S_{\kappa}$ contains a non-degenerate quadruple that is also isometrically embeddable in $\mathbb{R}^{2}$. (For the proof see [17], pp. 372-373).

that are not incident to $v$. Here $\overline{\operatorname{St}}(v)$ denotes the closed star of $v$, i.e. the smallest subcomplex (of the given simplicial complex $K$ ) that contains $\operatorname{St}(v)$, namely $\overline{\operatorname{St}}(v)=$ $\{\sigma \in \operatorname{St}(v)\} \cup\{\theta \mid \theta \leqslant \sigma\}$, where $\operatorname{St}(v)$ denotes the star of $v$, that is the set of all simplices that have $v$ as a face, i.e $\operatorname{St}(v)=\{\sigma \in K \mid v \leqslant \sigma\}$. 
(b) The quadruple $Q$ of distances $d_{13}=d_{14}=d_{23}=d_{24}=\pi, d_{12}=$ $d_{34}=3 \pi / 2$ admits exactly two embedding curvatures: $\kappa_{1}=\frac{1}{2}$ and $\kappa_{2} \in\left(\frac{1}{4}, \frac{4}{9}\right)$. (See [18].)

However, for "nice enough" metric spaces - i.e. spaces that are locally "plane like" - the embedding curvature exists and it is unique. Moreover and this represents a fact that is highly significative and quite relevant for our ends - this embedding curvature coincides with the classical Gaussian curvature.

Definition 3.33. A metric quadruple $Q=Q\left(p_{1}, p_{2}, p_{3}, p_{4}\right)$, of distances $d_{i j}=\operatorname{dist}\left(p_{i}, p_{j}\right), i=1, \ldots, 4$, is called semi-dependent (or a sd-quad, for brevity), iff 3 of its points are on a common geodesic, i.e. there exist 3 indices, e.g. $1,2,3$, such that: $d_{12}+d_{23}=d_{13}$.

One of the main advantages of sd-quads resides in the following result:

Proposition 3.34. An sd-quad admits at most one embedding curvature.

A classification criterion (due to Berestkovskii [11], see also [74], Theorem 18) for embedding curvature possibilities in the general case also exists:

Theorem 3.35. Let $M, Q$ be as above. Then one and only one of the following assertion holds:

1. $Q$ is linear.

2. $Q$ has exactly one embedding curvature.

3. $Q$ can be isometrically embedded in some $\mathcal{S}_{\kappa}^{m}, m \geq 2$; where $\kappa \in\left[\kappa_{1}, \kappa_{2}\right]$ or $\left(-\infty, \kappa_{0}\right]$, where $\mathcal{S}_{\kappa}^{m} \equiv \mathbb{R}^{m}$, if $\kappa=0 ; \mathcal{S}_{\kappa}^{m} \equiv \mathbb{S}_{\sqrt{\kappa}}^{m}$, if $\kappa>0$; and $\mathcal{S}_{\kappa}^{m} \equiv \mathbb{H}_{\sqrt{-\kappa}}^{m}$, if $\kappa<0$. Moreover, $m=2$ iff $\kappa \in\left\{\kappa_{0}, \kappa_{1}, \kappa_{2}\right\}$. (Here $\mathbb{S}_{\sqrt{\kappa}}^{m}$ denotes the $m$-dimensional sphere of radius $R=1 / \sqrt{\kappa}$, and $\mathbb{H}_{\sqrt{-\kappa}}^{m}$ stands for the $m$-dimensional hyperbolic space of curvature $\sqrt{-\kappa}$, as represented by the Poincaré model of the ball of radius $R=1 / \sqrt{-\kappa}$ ).

4. There exist no $m$ and $k$ such that $Q$ can be isometrically embedded in $\mathcal{S}_{\kappa}^{m}$.

3.2.2.1. Wald and Gauss curvatures comparison Again, as in the case of 1-dimensional metrizations of curvature (namely the Menger and Haantjes curvatures) one wishes to confirm that, indeed, Wald's curvature is a proper metrization of the classical (smooth) notion, and not just a mathematical "recreation", empty of any meaningful geometric content. In Applied Mathematics/Computer Science terms, one has to convince himself (and others!...) that this new, metric curvature can function as a discretization of the smooth notion. Fortunately, such a result does exists: 
Theorem 3.36 (Wald [118]). Let $S \subset \mathbb{R}^{3}, S \in \mathcal{C}^{m}, m \geq 2$ be a smooth surface. Then, given $p \in S, \kappa_{W}(p)$ exists and $\kappa_{W}(p)=K(p)$, where $K(p)$ denotes the Gaussian curvature at $p$.

In addition, Wald proved that the following partial reciprocal theorem also holds:

Theorem 3.37. Let $M$ be a compact and convex metric space. If $\kappa_{W}(p)$ exists, for all $p \in M$, then $\mathrm{M}$ is a smooth surface and $\kappa_{W}(p)=K(p)$, for all $p \in M$.

3.2.2.2. Computation of Wald curvature I: formula The allure of Wald curvature does not reside in its intuitive simplicity, but also that it comes endowed, so to say, with a simple formula for its actual computation: ${ }^{13}$

$$
\kappa(Q)=\left\{\begin{array}{cl}
0 & \text { if } D(Q)=0 \\
\kappa, \kappa<0 & \text { if } \operatorname{det}\left(\cosh \sqrt{-\kappa} \cdot d_{i j}\right)=0 ; \\
\kappa, \kappa>0 & \text { if } \operatorname{det}\left(\cos \sqrt{\kappa} \cdot d_{i j}\right) \text { and } \sqrt{\kappa} \cdot d_{i j} \leq \pi \\
& \text { and all the principal minors of order } 3 \text { are } \geq 0
\end{array}\right.
$$

where $d_{i j}=d\left(x_{i}, x_{j}\right), 1 \leq i, j \leq 4$, and $D(Q)$ denotes the so called CayleyMenger determinant:

$$
D\left(x_{1}, x_{2}, x_{3}, x_{4}\right)=\left|\begin{array}{ccccc}
0 & 1 & 1 & 1 & 1 \\
1 & 0 & d_{12}^{2} & d_{13}^{2} & d_{14}^{2} \\
1 & d_{12}^{2} & 0 & d_{23}^{2} & d_{24}^{2} \\
1 & d_{13}^{2} & d_{23}^{2} & 0 & d_{34}^{2} \\
1 & d_{14}^{2} & d_{24}^{2} & d_{34}^{2} & 0
\end{array}\right|
$$

The formula above might appear mystifying but, in fact, it has a very simple geometric justification based on the fact ${ }^{14}$ that

$$
D\left(p_{1}, p_{2}, p_{3}, p_{4}\right)=8\left(\operatorname{Vol}\left(p_{1}, p_{2}, p_{3}, p_{4}\right)\right)^{2},
$$

where $\operatorname{Vol}\left(p_{1}, p_{2}, p_{3}, p_{4}\right)$ denotes the (un-oriented) volume of the parallelepiped determined by the vertices $p_{1}, \ldots, p_{4}$ (and with edges $\overrightarrow{p_{1} p_{2}}, \overrightarrow{p_{1} p_{3}}, \overrightarrow{p_{1} p_{4}}$ ). This implies immediately

\footnotetext{
${ }^{13}$ This is in stark contrast with the Alexandrov (comparison) curvature (see below), at least in its usual presentation.

${ }^{14}$ proved by Cayley in his very first mathematical paper [27] (published while he was still making his living as a lawyer!...)
} 
Proposition 3.38. The points $p_{1}, \ldots, p_{4}$ are the vertices of a non-degenerate simplex in $\mathbb{R}^{3}$ iff $D\left(p_{1}, p_{2}, p_{3}, p_{4}\right) \neq 0$ :

Evidently, this means also that the opposite assertion also holds, namely that a simplex of vertices $p_{1}, \ldots, p_{4}$ is degenerate, i.e. isometrically embeddable in the plane $\mathbb{R}^{2} \equiv S_{0}$.

It is not hard deduce that the expressions appearing in Formula (3.40) for the cases where $\kappa \neq 0$ represent the equivalents of $D(Q)$ in the hyperbolic, respective spherical cases, using the well known fact, that, in the spherical (resp. hyperbolic) metric, the distances $d_{i j}$ are replaced by $\cos d_{i j}$ (resp. $\left.\cosh d_{i j}\right)$. However, the proof of this fact, as well for the analogous formulas and results in higher dimension are far too encumbering for this restricted exposition, so we have no choice but to advise the reader to consult [17].

Remark 3.39. A stronger result along these lines also exists. We formulate it - for convenience and practicality - for the case $n=3$, only. However it is readily generalized to any dimension. (For proofs and further details, see [17].)

Theorem 3.40. Let $d_{i j}>0,1 \leq i, j \leq 4, i \neq j$. Then there exists a simplex $\tau=T\left(p_{1}, \ldots, p_{4}\right) \subseteq \mathbb{R}^{3}$, such that $\operatorname{dist}\left(x_{i}, x_{j}\right)=d_{i j}, i \neq j ;$ iff $D\left(p_{i}, p_{j}\right)<$ $0,(\forall)\{i, j\} \subset\{1, \ldots, 4\}$ and $D\left(p_{i}, p_{j}, p_{k}\right)>0,(\forall)\{i, j, k\} \subset\{1, \ldots, 4\}$; where, for instance,

$$
D\left(p_{1}, p_{2}\right)=\left|\begin{array}{ccc}
0 & 1 & 1 \\
1 & 0 & d_{12}^{2} \\
1 & d_{12}^{2} & 0
\end{array}\right|
$$

and

$$
D\left(p_{1}, p_{2}, p_{3}\right)=\left|\begin{array}{cccc}
0 & 1 & 1 & 1 \\
1 & 0 & d_{12}^{2} & d_{13}^{2} \\
1 & d_{12}^{2} & 0 & d_{23}^{2} \\
1 & d_{13}^{2} & d_{23}^{2} & 0
\end{array}\right|
$$

etc...

It turns out that one can further relax the conditions of the previous theorem to obtain

Proposition 3.41. Let $d_{i j}>0,1 \leq i, j \leq 4, i \neq j$. Then there exists a simplex $\tau=\tau\left(p_{1}, \ldots, p_{4}\right) \subseteq \mathbb{R}^{3}$, such that $\operatorname{dist}\left(x_{i}, x_{j}\right)=d_{i j}, i \neq j$; iff $D\left(p_{1}, p_{2}, p_{3}, p_{4}\right) \neq 0$ and sign $D\left(p_{1}, p_{2}, p_{3}, p_{4}\right)=+1$.

In fact, one can generalize the results above to characterize the embedding curvature in terms of the angles of the model triangles. For this, we need first some further preliminaries: 

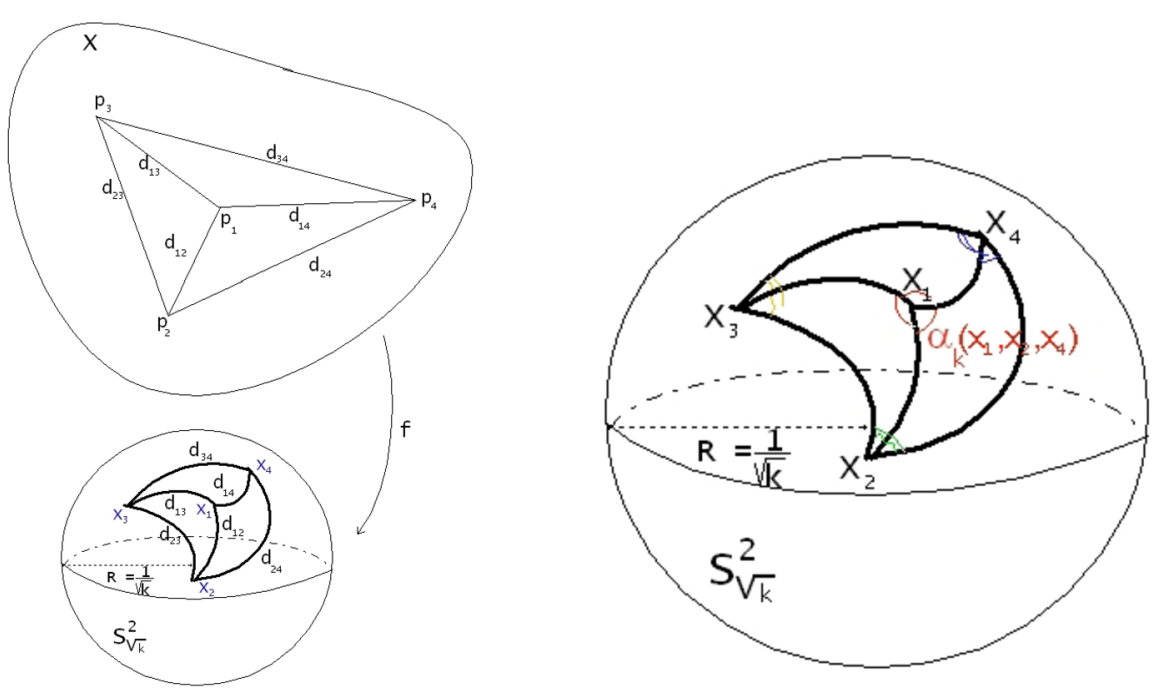

Figure 10: The angles $\alpha_{\kappa}\left(x_{i}, x_{j}, x_{l}\right)$ (right), induced by the isometric embedding of a metric quadruple in $\mathbb{S}_{\sqrt{\kappa}}^{2}$ (left).

Let $Q=\left\{x_{1}, x_{2}, x_{3}, x_{4}\right\}$ be a metric quadruple and let $V_{\kappa}\left(x_{i}\right)$ be defined as follows:

$$
V_{\kappa}\left(x_{i}\right)=\alpha_{\kappa}\left(x_{i} ; x_{j}, x_{l}\right)+\alpha_{\kappa}\left(x_{i} ; x_{j}, x_{m}\right)+\alpha_{\kappa}\left(x_{i} ; x_{l}, x_{m}\right)
$$

where $x_{i}, x_{j}, x_{l}, x_{m} \in Q$ are distinct, and $\kappa$ is any number (see Figure 10).

We can now state the sought characterization of Wald-Berestovskii in terms of angle sum:

Proposition 3.42 ([74], Theorem 23). Let $(X, d)$ be a metric space and let $U \in X$ be an open set. $U$ is a region of curvature $\geq \kappa$ iff $V_{\kappa}(x) \leq 2 \pi$, for any metric quadruple $\{x, y, z, t\} \subset U$.

Remark 3.43. The presence of angles in result above raises two issues regarding the role of angles in metric geometry:

1. From the practical (applications oriented) point of view, the proposition above shows that, in fact, the metric approach to curvature is essentially equivalent to the combinatorial (angle-based) one, as far as polyhedral surfaces (in $\mathbb{R}^{3}$ ) are considered. ${ }^{15}$ In particular, as far

${ }^{15}$ This holds, of course, up to the specific type of convergence for the metric and combinatorial curvature, i.e pointwise and in measure, respectively. 
as approximations of smooth surfaces in $\mathbb{R}^{3}$ are concerned, both approaches converge to the classical Gauss curvature.) Of course, the metric approach is far more general, since it is applicable to a very large class of metric spaces (see discussion at the end of this section).

2. From a more viewpoint, Proposition 3.42 shows that a "purely metric" (i.e. angle independent) approach is not truly possible for, even if "pure metric" formulas can be devised, the angles are also always "hidden in the substrate". (See also the discussion above regarding the applications of thick triangulations and metric curvatures to Regge calculus.) We might add that one can express (at least in the Euclidean case) the Cayley-Menger determinant in terms of (face or, alternatively, dihedral) angles incident to a vertex (see [17], p. 344).

3.2.2.3. Computation of Wald curvature II: approximation Unfortunately, using Formula (3.39) for the actual computation of $\kappa(Q)$ is highly problematic, since the equations involved are - apart from the Euclidean case - transcendental, therefore not solvable, in general, using elementary methods. In addition, they display certain numerical instability when solved using computer assisted methods. (See [80], [97] for a more detailed comments and some numerical results.)

We should also remark that Formula (3.39) implies that, in practice, a renormalization might be necessary for some of the vertices of positive Wald-Besetkovskii curvature, which represents yet another hindrance in it use.

It is therefore fortunate that a good approximation result, due to Robinson [79] exists. Not only does his result give a rational formula for approximating $\kappa(Q)$ and provide good error estimates, it also solves one other problem inherent in the use of the Wald curvature, namely the possible lack of uniqueness of the computed curvature. The way one circumvents this problem and the other pitfalls of Formula (3.39) is to make appeal to the simpler geometric configuration of sd-quads:

Theorem 3.44 ([79]). Given the metric semi-dependent quadruple $Q=$ $Q\left(p_{1}, p_{2}, p_{3}, p_{4}\right)$, of distances $d_{i j}=d\left(p_{i}, p_{j}\right), i, j=1, \ldots, 4$; the embedding curvature $\kappa(Q)$ admits a rational approximation given by:

$$
K(Q)=\frac{6\left(\cos \measuredangle_{0} 2+\cos \measuredangle_{0} 2^{\prime}\right)}{d_{24}\left(d_{12} \sin ^{2}\left(\measuredangle_{0} 2\right)+d_{23} \sin ^{2}\left(\measuredangle_{0} 2^{\prime}\right)\right)}
$$

where: $\measuredangle_{0} 2=\measuredangle\left(p_{1} p_{2} p_{4}\right), \measuredangle_{0} 2^{\prime}=\measuredangle\left(p_{3} p_{2} p_{4}\right)$ represent the angles of the Euclidian triangles of sides $d_{12}, d_{14}, d_{24}$ and $d_{23}, d_{24}, d_{34}$, respectively. 
Moreover the absolute error $R$ satisfies the following inequality:

$$
|R|=|R(Q)|=|\kappa(Q)-K(Q)|<4 \kappa^{2}(Q) \operatorname{diam}^{2}(Q) / \lambda(Q),
$$

where $\lambda(Q)=d_{24}\left(d_{12} \sin \measuredangle_{0} 2+d_{23} \sin \measuredangle_{0} 2^{\prime}\right) / S^{2}$, and where $S=\operatorname{Max}\left\{p, p^{\prime}\right\}$; $2 p=d_{12}+d_{14}+d_{24}, 2 p^{\prime}=d_{32}+d_{34}+d_{24}$.

Since we have presented in detail the proof of the theorem above both in [80] and in [97], we do not bring it here also, but rather refer the interested reader to either of these articles (and, of course, to Robinson's original paper [79]). We do, however, would like to underline that basic idea of the proof is to emulate, in a general metric setting, the original way of defining Gaussian curvature - in this case, rather than accounting for the area distortion, one measures the curvature by the amount of "bending" one has to apply to a general planar quadruple so that it may be "straightened" to a triangle $\triangle\left(p_{1} p_{3} p_{4}\right)$, with $p_{2}$ lying on the edge $p_{1} p_{3}$ - i.e. isometrically embedded as a $s d$-quad - in some $S_{\kappa}$.

Remark 3.45. In special cases (e.g. when $d_{12}=d_{32}$, etc.) simpler formulas are obtained en lieu of (3.43) - see [79], or [80], [97].

Of course, one is entitled to ask himself weather Formula (3.43) (or its variations mentioned above) is truly efficient in applications. The following example, due to Robinson himself, suggests that, at least in some cases, the actual computed error is far smaller then the one given by Formula (3.44).

Example 3.46 ([79]). Let $Q_{0}$ be the quadruple of distances $d_{12}=d_{23}=$ $d_{24}=0.15, d_{14}=d_{34}$ and of embedding curvature $\kappa=\kappa\left(Q_{0}\right)=1$. Then $\kappa S^{2}<1 / 16$ and $K\left(Q_{0}\right) \approx 1.0030280$, whereas the error computed using formula (3.44) is $|R|<0.45$.

For some experimental results and comparison to other metric curvatures for images, see [97]. We wish, however, to emphasize here that the results therein do comply to the expectations arising from the following (quite expected) theorem:

Theorem 3.47. Let $S$ be a smooth (differentiable) surface. Then, for any point $p \in S$ :

$$
K_{G}(p)=\lim _{n \rightarrow 0} K\left(Q_{n}\right) ;
$$

for any sequence $\left\{Q_{n}\right\}$ of sd-quads that satisfy the following conditions:

$$
Q_{n} \rightarrow Q=\square p_{1} p p_{3} p_{4} ; \operatorname{diam}\left(Q_{n}\right) \rightarrow 0 .
$$


Sketch of Proof. Recall that the Gaussian curvature $K_{G}(p)$ at a point $p$ is given by:

$$
K_{G}(p)=\lim _{n \rightarrow 0} \kappa\left(Q_{n}\right) ;
$$

where $Q_{n} \rightarrow Q=\square p_{1} p p_{3} p_{4} ; \operatorname{diam}\left(Q_{n}\right) \rightarrow 0$. But, if $Q$ is any sd-quad, then $\kappa^{2}(Q) \operatorname{diam}^{2}(Q) / \lambda(Q)<\infty$. Moreover, $|R|$ is small if $Q$ is not close to linearity. In this case $|R(Q)| \sim \operatorname{diam}^{2}(Q)$, for any given $Q$ (see [79]). The theorem now follows easily.

Remark 3.48. The convergence assured by Theorem 3.47 is not just in the sense of measures and errors of different signs do not simply cancel each other. Indeed, $\operatorname{sign}(\kappa(Q))=\operatorname{sign}(K(Q))$, for any metric quadruple $Q$.

3.2.2.4. Wald curvature and isometric embeddings Proposition 3.38 and Remark 3.39 rise the most general problem of the existence of isometric embeddings of metric metric spaces into gauge spaces. While in its full generality this is, of course, an untenable problem, we are interested in the much more restricted, but important in our context (Graphics, Imaging, Mathematical Modeling, etc.), problem of isometric embedding of $P L$ surfaces in $\mathbb{R}^{3}$.

A first result in this direction is a criterion for the local isometric embedding of polyhedral surfaces in $\mathbb{R}^{3}$, akin to the classical Gauss fundamental (compatibility) equation in the classical differential geometry of surfaces, that we first proved in [90]. Namely, given a vertex $v$, with metric curvature $K_{W}(v)$, the following system of inequalities should hold:

$$
\left\{\begin{array}{l}
\max A_{0}(v) \leq 2 \pi \\
\alpha_{0}\left(v ; v_{j}, v_{l}\right) \leq \alpha_{0}\left(v ; v_{j}, v_{p}\right)+\alpha_{0}\left(v ; v_{l}, v_{p}\right), \text { for all } v_{j}, v_{l}, v_{p} \sim v \\
V_{\kappa}(v) \leq 2 \pi
\end{array}\right.
$$

Here

$$
A_{0}=\max _{i} V_{0} ;
$$

" $\sim$ " denotes incidence, i.e. the existence of a connecting edge $e_{i}=v v_{j}$ and, of course, $V_{\kappa}(v)=\alpha_{\kappa}\left(v ; v_{j}, v_{l}\right)+\alpha_{\kappa}\left(v ; v_{j}, v_{p}\right)+\alpha_{\kappa}\left(v ; v_{l}, v_{p}\right)$, where $v_{j}, v_{l}, v_{p} \sim v$, etc.

Note that the first two inequalities represent the (extrinsic) embedding condition, while the third one represents the intrinsic curvature (of the $P L$ manifold) at the vertex $v$.

For details and a fitting global embedding criterion see [90]. 
Remark 3.49. Possible applications include those fields where isometric embedding of finite metric spaces is relevant, such as Communication Networks, Graphics Imaging and Vision, Elastic membranes, Protein folding, Genetics (e.g when functional distance between genes is of interest) and Manifold Learning.

Remark 3.50. In Formula 3.45 the correct definition of the open neighbourhood of a vertex is essential (see the relevant discussion in Section 1).

Remark 3.51. As far as the possible (non-theoretical) applications of Wald curvature are concerned, one could define (see [94]), in analogy with the Menger curvature measure (see (3.28) above) a Wald curvature measure:

$$
\mu_{W}(v)=K_{W}(v) \cdot \operatorname{Area}(S t(v)),
$$

where $S t(v)$ denotes the star of the vertex $v$.

The applications of the Wald measure would be similar to those of the Meger measure, with the potential for uses in more general settings (e.g. cloud of points).

\subsection{Higher dimensions I: basic definitions}

The treatment of metric curvature in higher dimensions should, if we followed the same logic as above, belong here. However, this does not represent a classical "state of the art", but it rather covers new developments that represent (mostly) our(the authors) own ideas and results.

In higher dimensions there is far less clear what "curvature" should be (or mean); we shall presently begin to understand wherefrom the problems stems. In consequence, we can't even begin to hope that we can make an exhaustive list of curvatures. Therefore we bring below only the mere essentials (that also prove to be the most important for the understanding of phenomenology in dimension $\geq 3$ ). Still, apart from these, we shall encounter later on yet another curvature (or rather another family of curvatures). However, these will appear only as an application of basic ideas and the tools we introduce in this section (and the following ones).

The most natural and intuitive idea to define curvature of higher dimensional manifolds would be, of course, to try and mimic the principal curvatures approach to Gaussian curvature. More precisely, one would be tempted to look at the Gaussian curvatures of all 2-dimensional sections of an $n$-dimensional manifold. Unfortunately, there are $n$ principal curvatures, and the symmetric polynomials do not convey sufficient (nor simple enough) geometric information. Moreover, even after symmetries reduce this 
to "only" $n^{2}\left(n^{2}-1\right) / 12$, for $n \geq 3$ there are clearly to many numbers to deal with and a simple, geometric interpretation is highly needed. Even apart from the considerations above, this shouldn't come as great surprise: Recall that Gauss revolutionary idea was to define curvature via the normal mapping and area, in order to avoid the very pitfalls of "sections" by normal planes, and only proved Formula 3.30 as a consequence of his far reaching definition.

While we shall see shortly that not everything is lost in this approach, for more theoretically robust definitions we need the (quite technical) notion of (Riemannian) curvature tensor. However, before defining it, we have to introduce some notations:

In the reminder of this section, $M^{n}$ denotes an $n$-dimensional $\mathcal{C}^{k}$-Riemannian manifold, $k \geq 2$, which we may presume, by Nash's theorem [66], to be isometrically embedded in $R^{N}$, for some $N$ sufficiently large. Analogously to the notation for surfaces, let $T_{p}\left(M^{n}\right)$ denote the tangent space at the point $p \in M^{n}$, and let $T_{p}^{\perp}\left(M^{n}\right)$ stand be the orthogonal complement of $T_{p}\left(M^{n}\right)$ in $T_{p}\left(\mathbb{R}^{N}\right)$, i.e. $T_{p}\left(M^{n}\right) \oplus T_{p}^{\perp}\left(M^{n}\right)=T_{p}\left(\mathbb{R}^{N}\right)$. Then $M^{n}$ can be locally written as the graph of a function $f: T_{p}\left(M^{n}\right) \rightarrow T_{p}^{\perp}\left(M^{n}\right)$.

\subsubsection{The curvature tensor}

Definition 3.52. Let $M^{n}$ and $f$ be as above, and let $p \in M^{n} \subset \mathbb{R}^{N}$. The bilinear form $I I_{p}: T_{p}\left(M^{n}\right) \rightarrow T_{p}^{\perp}\left(M^{n}\right)$

$$
I I_{p}\left(M^{n}\right)=\left(\beta_{i j}\right)_{1 \leq i, j \leq n}
$$

where $\beta_{i j}=\partial^{2} f / \partial x_{i} \partial x_{j}, 1 \leq i, j \leq n$; is called the second fundamental tensor of $M^{n}$ at the point $p$.

The Riemannian curvature tensor (at a point $p$ ) is defined as the tensor of $2 \times 2$-minors of $I I_{p}\left(M^{n}\right)$, i.e.:

$$
R_{i j k l}=\beta_{i k} \beta_{j l}-\beta_{j k} \beta_{i l} .
$$

Remark 3.53. Riemannian curvature is not intrinsic.

It is quite probable that the reader is already convinced that, in the words of Gromov, "the curvature tensor of a Riemannian manifold is a little monster of (multi)linear algebra whose full geometric meaning remains obscure" ([42], p.10). If not yet, then its further appearances in the sequel will persuade him that the need for more geometric methods is quite stringent. 
3.3.2. Sectional curvature The sections approach to curvature mentioned above is encoded in the notion of the (quite properly and evidently called) sectional curvature:

Definition 3.54. Let $p \in M^{n}$, and let $\Pi \subset T_{p}\left(M^{n}\right)$ be a 2-dimensional plane, and let $S=M^{n} \cap\left(\Pi \oplus T_{p}^{\perp}\left(M^{n}\right)\right)$. Then $\operatorname{dim} S=2$ and we define $K(\Pi)=K_{p}(S)$, where $K_{p}(S)$ represents the Gauss curvature of $S$ at the point $p$.

Of course, if $n=2, K$ reduces to the classical Gauss curvature, thus fully justifying not only its name, but also its the notation.

We should make clear that we didn't introduce the curvature tensor for not: Indeed, there is a close connection between sectional curvature and the Riemannian curvature tensor, as can be seen from the following formula:

$$
K(\Pi)=\sum_{1 \leq i, j, k, l \leq n} R_{i j k l} \mathbf{x}_{\mathbf{i}} \mathbf{x}_{\mathbf{j}} \mathbf{x}_{\mathbf{k}} \mathbf{x}_{\mathbf{l}} ;
$$

where $\left\{\mathbf{x}_{h}\right\}_{1 \leq h \leq n}$ is an orthonormal base of $T_{p}\left(M^{n}\right)$.

Moreover, it is easy to show by direct computations that knowledge of $K(M)$ on all tangent planes is equivalent to knowing the curvature tensor (see, e.g. [34], pp. 94-95). This shows why the sectional approach to curvature (in dimension $\geq 3$ ) leads, at least in some sense, to a "geometrical deadend": it is essentially equivalent to Gromov's algebraic "little monster"...

Given the very definition of sectional curvature, it will come as no surprise that an analogue (and slight generalization) of the Bertrand-DiguetPuiseaux formula (3.36) is given by:

\section{Proposition 3.55.}

$$
\text { length } C(p, \varepsilon, \alpha)=\alpha \varepsilon-\frac{\alpha}{3 \sin ^{2} \alpha} K(p) \varepsilon^{3}+o\left(\varepsilon^{3}\right),
$$

where $C(p, \varepsilon, \alpha)$ denotes the arc of length $\alpha$ of $C(p, \varepsilon)$. In particular, for $\alpha=2 \pi$ (and $n=2$ ) one gets the Bertrand-Diguet-Puiseaux formula in its classical form.

Thus sectional curvature (hence the curvature tensor) measure the defect of $M^{n}$ from being locally Euclidean. This is done at the 2-dimensional level as a measure of the divergence of two geodesics starting from a common point, as a function of the initial angle made by the geodesics (or, equivalently, by their tangents at the starting point). In fact, $M^{n}$ is flat (i.e. locally Euclidean) iff $K \equiv 0$. In addition, if $K \equiv k_{0}$, where $k_{0}$ is a constant, then 


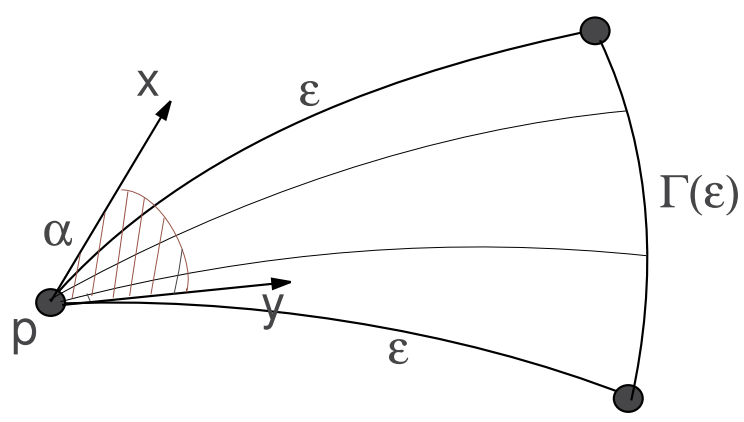

Figure 11: The geometric interpretation of sectional curvature ensuing from the Bertrand-Diguet-Puiseaux: Sectional curvature measures the divergence rate of two geodesics staring from a common point, as a function of the initial angle made by the geodesics. (After [12].)

$M^{n}$ is locally isometric to the simply connected space of constant sectional curvature.

Remark 3.56. Recall the adagio equating curvature with second derivative. Given the analogous fact for the Gaussian curvature, it is easy to see that, in fact, $K$ behaves like $a$ second derivative - more precisely as the Hessian of the metric $g$ (see [13], p. 267).

Moreover, if one is willing to restrict himself to the scope of "testing", another more flexible and extremely useful notion of curvature presents itself:

3.3.3. Ricci curvature Formally Ricci curvature is defined as the contraction of the Riemannian curvature tensor:

Definition 3.57. Let $\mathbf{v} \in T_{p} M^{n}$ be a unit vector. The Ricci curvature in the direction $\mathbf{v}$ is defined (in local coordinates) as:

$$
\operatorname{Ric}_{i j}=\sum_{i} R_{i j i l}
$$

From the definition above and from the relationship between the Riemannian curvature tensor and sectional curvature (see above), it follows that:

$$
\operatorname{Ric}(\mathbf{v})=\operatorname{Ric}(\mathbf{v}, \mathbf{v})=\sum_{i=2}^{n} K\left(\mathbf{v}, \mathbf{x}_{i}\right)=\sum_{i=2}^{n} R\left(\mathbf{v}, \mathbf{x}_{i}, \mathbf{v}, \mathbf{x}_{i}\right),
$$

where $\left\{\mathbf{v}, \mathbf{x}_{1}, \ldots, \mathbf{x}_{n-1}\right\}$ represents an orthonormal base of $T_{p} M^{n}$. 


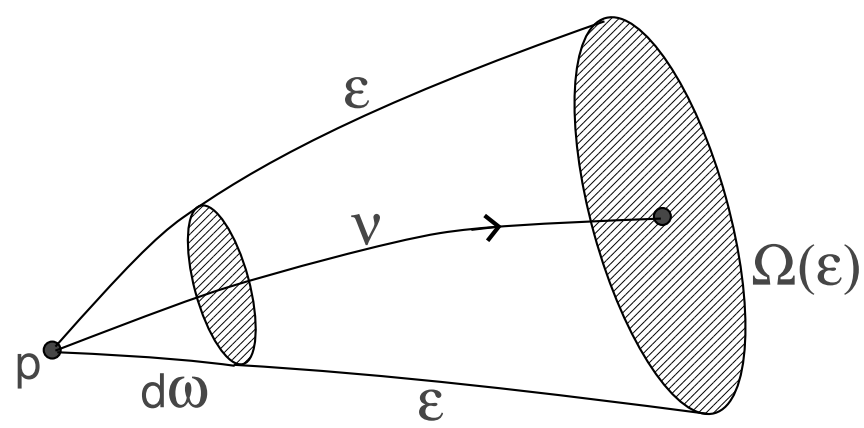

Figure 12: Ricci curvature as defect of the manifold from being locally Euclidean in various tangential directions (after [12]). Here $d \omega$ denotes the $n$-dimensional solid angle in the direction of the vector $\mathbf{v}, \Omega(\alpha)$ the $(n-1)$ volume generated by geodesics of length $\varepsilon$ in $d \omega$, and $\operatorname{Ric}(\mathbf{v})$ the Ricci curvature in the direction $\mathbf{v}$.

Moreover, let $\Pi=<\mathbf{v}, \mathbf{w}>$ denote the plane spanned by $\mathbf{v}$ and $\mathbf{w}$. Then the following holds:

$$
\mathbf{v} \cdot \operatorname{Ric}(\mathbf{v})=\frac{n-1}{\operatorname{Vol}\left(\mathbb{S}^{n-2}\right)} \int_{\mathbf{w} \in T_{p}\left(M^{n}\right), \mathbf{w} \perp \mathbf{v}} K(<\mathbf{v}, \mathbf{w}>) ;
$$

that is the Ricci curvature represents an average of sectional curvatures.

Remark 3.58. The curvature-as-second-derivative leitmotif has its Ricci "avatar" so to say, too. Without entering into the technical details, one should keep in mind that Ricci curvature "should be thought as the Laplacian of $g$ ". ${ }^{16}$

Theorem $3.59([54])$. Let $M^{n}$ be as above. Denote by d $\alpha$ the $n$-dimensional solid angle in the direction of the vector $\mathbf{v} \in T_{p}\left(M^{n}\right)$ and by $\omega(\alpha)$ the $(n-1)$ volume generated by geodesics of length $\varepsilon$ in $d \alpha$. Then:

$$
\operatorname{Vol}(\omega(\alpha))=d \alpha \varepsilon^{n-1}\left(1-\frac{\operatorname{Ric}(\mathbf{v})}{3} \varepsilon^{2}+o\left(\varepsilon^{2}\right)\right) .
$$

Remark 3.60. See also [43] (pp. 316-319), [40], [116], for various generalization of the result above. Also, for applications see [46], [116], [86].

3.3.4. Scalar curvature We conclude with the least flexible (and expressive) of the basic, essential curvatures, namely the scalar curvature. This is defined as the trace of the Ricci curvature:

\footnotetext{
${ }^{16}[73]$, p.30. For further technical details see, e.g., [73].
} 
Definition 3.61 (Scalar Curvature).

$$
\text { scal }=\sum_{i} \operatorname{Ric}_{i i}
$$

It follows immediately from the definition that, for any orthonormal basis $\left\{\mathbf{x}_{1}, \ldots, \mathbf{x}_{n}\right\}$ of $T_{p}\left(M^{n}\right)$, the following analogue of Formula (3.54) holds:

$$
\text { scal }=2 \sum_{1 \leq i<j \leq n} K\left(<\mathbf{x}_{i}, \mathbf{x}_{j}>\right)=\frac{n(n-1)}{\operatorname{Vol}(\Pi)} \int_{\Pi \in \mathcal{P}} K(\Pi) ;
$$

where $\mathcal{P}$ represents the collection of 2 -planes in $T_{p}\left(M^{n}\right)$.

Note that, as the name suggests, scalar curvature is, indeed, a scalar (as opposed to sectional and Ricci curvatures that are tensors). However, it is important to make the following observation, not least because we shall make appeal to it in the sequel:

Remark 3.62. In dimension $n=2$ all the curvatures above (sectional, Ricci, scalar) essentially coincide and, in fact, so do, in dimension, $n=3$, Ricci curvature and sectional curvature. (More precisely formulas can be found, for instance, in [73], [113].)

As expected, this curvature also admits an analogue of the second Bertrand-Diguet-Puiseaux formula:

Theorem 3.63 ([38], p. 166).

$$
\operatorname{Vol} B(p, \varepsilon)=\omega_{n} \varepsilon^{n}\left(1-\frac{1}{6(n+2)} \operatorname{scal}(p) \varepsilon^{2}+o\left(\varepsilon^{2}\right)\right) ;
$$

where $\omega_{n}$ denotes the volume of the unit ball in $\mathbb{R}^{n}$.

That is, scalar curvature measures the defect of the manifold from being locally Euclidean at the level of volumes of small geodesic balls.

\subsection{Higher dimensions II: Lipschitz-Killing curvatures}

Given a Riemannian manifold $M^{n}$, the Lipschitz-Killing curvatures of $M^{n}$ are defined as follows:

$$
\begin{gathered}
R^{j}\left(M^{n}\right)=\frac{1}{(n-j) ! 2^{j} \pi^{j / 2}(j / 2) !} \sum_{\pi \in S_{n}}(-1)^{\epsilon(\pi)} \Omega_{\pi(1) \pi(2)} \wedge \cdots \wedge \Omega_{\pi(j-1) \pi(j)} \wedge \\
\wedge \omega_{\pi(j+1)} \wedge \cdots \wedge \omega_{\pi(n)},
\end{gathered}
$$


where $\Omega_{\pi(j-1) \pi(j)}$ are the curvature 2 -forms and $\omega_{k l}$ denote the connection 1 -forms, and they are interrelated by the structure equations:

$$
\left\{\begin{array}{l}
d \omega_{k}=-\sum_{i} \omega_{k l} \wedge \omega_{l}, \\
d \omega_{k l}=-\sum_{i} \omega_{k i} \wedge \omega_{i l}+\Omega_{k l} .
\end{array}\right.
$$

where $\left\{\omega_{k}\right\}$ is the dual basis of $\left\{e_{k}\right\}$. Moreover, the integral $\int_{M^{n}} R^{j}$ is also known as the integrated mean curvature (of order $j$ ).

Remark 3.64. While the expression (3.59) above might appear daunting ${ }^{17}$, in low dimensions Lipschitz-Killing curvatures are, in fact, quite familiar: $R^{0} \equiv$ volume and $R^{2} \equiv$ scalar curvature. Moreover, $R^{n} \equiv$ Gauss-Bonnet-Chern form, (for $n=2 k$ ).

We also have the following expression of the Lipschitz-Killing curvatures: (3.61)

$$
R^{j}\left(M^{n}\right)=\frac{1}{\operatorname{Area}\left(\mathbb{S}^{n-j-1}\right)} \int_{M^{n-1}} S_{n-j-1}\left(k_{1}(x), k_{2}(x), \ldots, k_{n-1}(x)\right) d \mathcal{H}^{n-1},
$$

where $M^{n-1}=\partial M^{n}, d \mathcal{H}^{n-1}$ denotes the $(n-1)$-dimensional Hausdorff measure, and where the symmetric functions $S_{j}$ are defined by:

$$
S_{j}\left(k_{1}(x), k_{2}(x), \ldots, k_{j-1}(x)\right)=\sum_{1 \leq k_{i_{1}} \leq k_{i_{k}} \leq j-1} k_{i_{1}}(x) \cdots k_{i_{k}}(x),
$$

$k_{1}(x), k_{2}(x), \ldots, k_{n-1}(x)$ being the principal curvatures - see e.g. [121].

Remark 3.65. Formula 3.61 above shows why the Lipschitz-Killing curvatures are also called the total mean curvatures (and the " $S_{j}$ "-s are called the mean curvatures (of order $j$ ). It also suggests a quite direct method of obtaining a local (point-wise) version.

In a similar manner (but technically slightly more complicated), one can define the associated boundary curvatures (or mean curvatures) $H^{j}$ which are curvature measures on $\partial M^{n}$ : Let $\left\{e_{k}\right\}_{1 \leq k \leq n}$ be an orthonormal frame for the tangent bundle $T_{M^{n}}$ of $M^{n}$, such that, along the boundary $\partial M^{n}$, $e_{n}$ coincides with the inward normal. Then, for any $2 k+1 \leq j \leq n$, we define

$$
H^{j}=\sum_{k} \Omega_{j, k}
$$

\footnotetext{
${ }^{17}$ and rightly so!...
} 
where

$$
\begin{gathered}
\Omega_{j, k}=c_{j, k} \sum_{\pi \in S_{n-1}}(-1)^{\epsilon(\pi)} \Omega_{\pi(1) \pi(2)} \wedge \cdots \wedge \Omega_{\pi(2 k-1) \pi(2 k)} \wedge \omega_{\pi(2 k+1), n} \wedge \cdots \\
\cdots \wedge \omega_{\pi(j-1), n}, \omega_{\pi(j)} \wedge \cdots \wedge \omega_{\pi(n-1)},
\end{gathered}
$$

and

$$
c_{j, k}= \begin{cases}(-1)^{k}\left(2^{j} \pi^{\frac{j-1}{2}} k !\left(\frac{j-1}{2}-k\right) !(n-j) !\right)^{-1}, & j=2 p+1 \\ (-1)^{k}\left(2^{k+\frac{j}{2}} \pi^{\frac{j}{2}} k !(j-2 k-1) !(n-j) !\right), & j=2 p .\end{cases}
$$

These curvatures measures are normalized by imposing the condition that:

$$
\int_{T^{n-j} \times M^{j}} R^{j}+\int_{T^{n-j} \times \partial M^{j}} H^{j}=\chi\left(M^{j}\right) \operatorname{Vol} T^{n-j},
$$

for any flat $T^{n-j}$.

Remark 3.66. As expected, in view of the similar elucidation of the LipschitzKilling curvatures, the low dimensional boundary curvatures also have quite familiar interpretations: $H^{1} \equiv$ area boundary, $H^{2} \equiv$ mean curvature for inward normal (as expected given the generic names for these $H^{j}$-s), etc.

\section{Thick triangulations and the approximation of curvatures}

This section is dedicated to a notion that appears, under many guises and in different - covert as well as overt - manners, again and again in many theoretical and applied settings, both in Mathematics (e.g. in Quasiregular Mappings Theory and Geometric Measure Theory), as well as Graphics and Imaging, namely that of "thick triangulation", and (some of) its connections with Metric Differential Geometry. (For applications in Geometric Function Theory, see e.g. [115], [71], [84], [86] and, for the relationship between fat triangulations, Geometric Function Theory and Discrete Differential Geometry, see [105], [98].)

\subsection{Thick triangulations}

We begin with the basic definition, as introduced in [29]: 
Definition 4.1. Let $\tau \subset \mathbb{R}^{n} ; 0 \leq k \leq n$ be a $k$-dimensional simplex. The thickness $\varphi$ of $\tau$ is defined as being:

$$
\varphi=\varphi(\tau)=\inf _{\substack{\sigma \leqslant \tau \\ \operatorname{dim} \sigma=j}} \frac{\operatorname{Vial}_{j}(\sigma)}{\operatorname{diam}^{j} \sigma} .
$$

The infimum is taken over all the faces of $\tau$ (including $\tau$ itself), $\sigma \leqslant \tau$, and $\operatorname{Vol}_{j}(\sigma)$ and diam $\sigma$ stand for the Euclidian $j$-volume and the diameter of $\sigma$ respectively. (If $\operatorname{dim} \sigma=0$, then $\operatorname{Vol}_{j}(\sigma)=1$, by convention.) A simplex $\tau$ is $\varphi_{0}$-thick, for some $\varphi_{0}>0$, if $\varphi(\tau) \geq \varphi_{0}$. A triangulation (of a submanifold of $\left.\mathbb{R}^{n}\right) \mathcal{T}=\left\{\sigma_{i}\right\}_{i \in \mathbf{I}}$ is $\varphi_{0}$-thick if all its simplices are $\varphi_{0}$-thick. A triangulation $\mathcal{T}=\left\{\sigma_{i}\right\}_{i \in \mathbf{I}}$ is thick if there exists $\varphi_{0} \geq 0$ such that all its simplices are $\varphi_{0}$-thick.

As already noted, the definition of fatness given above is that introduced in [29]. One reason for doing this is to preserve the "unity of style", so to say: since we heavily rely, at least in the first part, on the results and techniques of [29], we find only fitting that we used, at least in the beginning, the same definition as that of Cheeger et al. However, they also prove in the same paper the following result that gives a more intuitive interpretation on the notion of thickness of simplices as a function of their dihedral angles in all dimensions:

Proposition $4.2([29])$. There exists a constant $c(k)$ that depends solely upon the dimension $k$ of $\tau$ such that

$$
\frac{1}{c(k)} \cdot \varphi(\tau) \leq \min _{\sigma<\tau} \measuredangle(\tau, \sigma) \leq c(k) \cdot \varphi(\tau),
$$

and

$$
\varphi(\tau) \leq \frac{\operatorname{Vol}_{j}(\sigma)}{\operatorname{diam}^{j} \sigma} \leq c(k) \cdot \varphi(\tau) .
$$

Here $\measuredangle(\tau, \sigma)$ denotes the (internal) dihedral angle. While intuitively simple, the formal definition ${ }^{18}$ of this notion requires some technical preliminaries:

Definition 4.3. A simplicial cone $C^{k} \subset \mathbb{R}^{k} \subset \mathbb{R}^{n}$, is defined as: $C^{k}=\bigcap^{k} H_{j}$, where $H_{j}$ are open half spaces in general position, such that $0 \in \stackrel{j=1}{H}{ }_{j}, j=$ $1, \ldots, k$.

$L^{k-1}=C^{k} \cap \mathbb{S}^{n-1}$ is called a spherical simplex.

\footnotetext{
${ }^{18}$ For an alternative definition, see [109] IV. 2, IX. 15.
} 
Definition 4.4. Consider the simplices $\sigma^{k}<\tau^{m}$, and let $p \in \sigma^{k}$. Define the normal cone: $C^{\perp}\left(\sigma^{k}, \tau^{m}\right)=\left\{\vec{p} \vec{x} \mid x \in \tau^{m}, \vec{p} \vec{x} \perp \sigma^{k}\right\}$, where $\overrightarrow{p x}$ denotes the ray through $x$ and base-point $p$.

The spherical simplex $L\left(\sigma^{k}, \tau^{m}\right)$ associated to $C^{\perp}\left(\sigma^{k}, \tau^{m}\right)$ is called the link of $\sigma^{k}$ in $\tau^{m}$.

Remark 4.5. $C^{\perp}\left(\sigma^{k}, \tau^{m}\right)$ does not depend upon the choice of $p$.

We are now to formally define the notion of dihedral angle, as follows:

Definition 4.6. The (internal) dihedral angle $\measuredangle\left(\tau^{k}, \sigma^{m}\right)$ of $\sigma$ in $\tau$ is the normalized volume of $L\left(\sigma^{k}, \tau^{m}\right)$, where the normalization is such that the volume of $\mathbb{S}^{n-1}$ equals 1 , for any $n \geq 2$.

A few remarks are mandatory at this point: Note first that condition (4.2) is just the expression of thickness as a function of dihedral angles in all dimensions. This fact warrants a number of observations:

- Using the dihedral angle approach in assuring the "aspect ratio" of a triangular mesh is commonly used in Computational Geometry, Computer Graphics and related fields, to ensure that the constituting tetrahedra are not to "flat" or too "slim", in particular that no "slivers", appear (see, e.g. [4], [32], [35]).

- The condition under scrutiny shows that thickness is hierarchical, in the sense that for a simplex to be thick, all its lower dimensional faces have to be thick. This is also transparent from condition 4.3 and we shall discuss this fact again shortly, from a different point of view.

- The dihedral angle definition appears to be very promising in view of some quite recent developments (e.g. [5], [10], [33], [78]) of Regge calculus [77]. Indeed, it seems that, from the Theoretical Physics, it has quite a number of advantages. However, it falls short from Regge's original goal, as stated in [77], for a purely metric gravity (both classical and quantum). Moreover, from the mathematicians point of view it also lacks the kind of "symmetry" one usually strives for, since it makes appeal to both angles and distances (e.g. in the "fattening" technique (using $\varepsilon$-moves) - see [29]). More importantly, one strives for the most general possible setting, thus one looks for the possible extensions of the results of [29] (and, in consequence, of the present paper) to as "general" metric spaces. Therefore, a purely metric approach for all aspects of the problem, including fatness, is desirable and we shall discuss it in detail below. 
Similarly, condition (4.3) expresses fatness as given by "large area/ diameter" (or "volume/diameter") ratio. Diameter is important since fatness is independent of scale. Note, again, the hierarchic property of fatness as expressed by this condition.

Moreover, even the defining condition of thickness (4.1) lacks this purely metric aspect. Indeed, while volume and edge lengths of a Euclidean simplex (or, for that matter, in any space form) are closely related, in a general metric space no volume is apriorily postulated. (Admittedly, one can attempt a fitting definition for metric measure spaces [86], but this has only limited purely mathematical applications and, furthermore, it also departs from the purely metric approach. ${ }^{19}$ ) Fortunately, this fault is easy to amend, using the so generalized ( $n$-dimensional) Cayley-Menger determinant, that expresses the volume of the $n$-dimensional Euclidean simplex $\sigma_{n}\left(p_{0}, p_{1}, \ldots, p_{n}\right)$ of vertices $p_{0}, p_{1}, \ldots, p_{n}$ as a function of its edges $d_{i j}, 0 \leq i<j \leq n$ :

$$
D\left(p_{0}, p_{1}, \ldots, p_{n}\right)=\left|\begin{array}{ccccc}
0 & 1 & 1 & \cdots & 1 \\
1 & 0 & d_{01}^{2} & \cdots & d_{1 n}^{2} \\
1 & d_{10}^{2} & 0 & \cdots & d_{1 n}^{2} \\
\vdots & \vdots & \vdots & \ddots & \vdots \\
1 & d_{n 0}^{2} & d_{n 1}^{2} & \cdots & 0
\end{array}\right| ;
$$

namely

$$
\operatorname{Vol}^{2}\left(\sigma_{n}\left(p_{0}, p_{1}, \ldots, p_{n}\right)\right)=\frac{(-1)^{n+1}}{2^{n}(n !)^{2}} D\left(p_{0}, p_{1}, \ldots, p_{n}\right)
$$

(Similar expressions for the Hyperbolic and Spherical simplex also exist, see, e.g. [17], [18]. However, we do not bring them here, not least because they are far too technical for this limited exposition; suffice therefore to add that they essentially reproduce the proof given in the Euclidean case, taking into account the fact that, when performing computations in the spherical (resp. hyperbolic) metric, one has to replace the distances $d_{i j}$ by $\cos d_{i j}$ (resp. $\cosh d_{i j}$ ) - see [18] for the full details.)

Evidently, the Cayley-Menger determinant makes sense for any metric $(n+1)$-tuple, i.e. for any metric metric space with $n+1$ points $p_{0}, p_{1}, \ldots, p_{n}$ and mutual distances $d_{i j}, 0 \leq i \leq j \leq n$.

As implicitly mentioned above, the definition of thickness as given by Definition 4.1 is only one of the (many) existing ones. An alternative one,

\footnotetext{
${ }^{19}$ even if such an approach is, in the light of the literature mentioned above, less useful in Physics than previously believed
} 
given solely in terms of distances, is given in [115]. Tukia's fatness ${ }^{20}$ of a simplex $\sigma=\sigma\left(p_{0}, \ldots, p_{n}\right)$ can be written as

$$
\varphi_{T}(\sigma)=\max _{\pi} \frac{\delta\left(p_{0}, \ldots, p_{n}\right)}{\operatorname{diam} \sigma},
$$

where $\delta=\delta\left(p_{0}, \ldots, p_{n}\right)=\operatorname{dist}\left(p_{i}, F_{i}\right)$, where $F_{i}$ denotes the $(n-1)$-dimensional face (of $\sigma$ ) opposite to $p_{i}$, and where the maximum is taken over all the permutations $\pi$ of $0, \ldots, n$. In other words, $\delta$ represents the maximal height $h_{\text {Max }}$ from the vertices of $\sigma$ to its $(n-1)$-dimensional faces (or facets). Note that this definition of thickness can also be used, for instance, for Hyperbolic simplices (as, indeed, Tukia did).

Since the diameter of a simplex is nothing but the longest edge $l_{\text {Max }}$, that is $\operatorname{diam} \sigma=\max _{0 \leq i<j \leq n} d_{i j}=\max _{1 \leq m \leq n(n+1) / 2} l_{m}$, Tukia's definition of thickness is, clearly, purely metric. It is true that the expression of $\varphi_{T}$ is not given solely in terms of the distances between the vertices (of $\sigma$ ), however it is easy to remedy this by computing $h_{i}$ - the distance from $p_{i}$ to the face $F_{i}$, using the classical and well known formula:

$$
\operatorname{Vol}(\sigma)=\frac{1}{n} h_{i} \operatorname{Area}\left(F_{i}\right)
$$

and then expressing the volume of $F_{i}$ using the fitting Cayley-Menger determinant, where we denoted the $(n-1)$-volume by "Area". (One has to proceed somewhat more carefully for the case, say, of Hyperbolic simplices, but we are not concerned here with this case.)

Peltonen's definition - see [71] - is, perhaps the easiest to express, even not if the simplest for actual computation, as she defines fatness (of a simplex) as:

$$
\varphi_{P}(\sigma)=\frac{r}{R},
$$

where $r$ denotes the radius of the inscribed sphere of $\sigma$ (inradius) and $\mathrm{R}$ denotes the radius of the circumscribed sphere of $\sigma$ (circumradius). The problem with this approach is that it is not given - explicitly, that is in terms of the lengths of the edges of the simplex. Again, this does not represent a serious inconvenience, since, given a simplex $\sigma=\sigma\left(p_{1}, \ldots, p_{n}\right)$,

\footnotetext{
${ }^{20}$ In fact he defines the reciprocal quantity which he calls flatness and denotes by $F(\sigma)$.
} 
the circumradius $R$ is given by the following formula:

$$
R=-\frac{1}{2} \frac{\Delta\left(p_{1}, \ldots, p_{n}\right)}{D\left(p_{1}, \ldots, p_{n}\right)}
$$

where

$$
\Delta\left(p_{1}, \ldots, p_{n}\right)=\left|\begin{array}{cccc}
0 & d_{12}^{2} & \cdots & d_{1 n}^{2} \\
d_{21}^{2} & 0 & \cdots & d_{1 n}^{2} \\
\vdots & \vdots & \ddots & \vdots \\
d_{n 1}^{2} & d_{n 2}^{2} & \cdots & 0
\end{array}\right|
$$

Moreover, the inradius $r$ can be computed quite simply using the fact (akin to formula (4.7)) that

$$
\operatorname{Vol} \sigma_{n}=\frac{1}{n} \sum_{1}^{n+1} \operatorname{Area}\left(F_{i}\right),
$$

and expressing again Area $\left(F_{i}\right)$ as a function of its edges.

On the other hand, the equivalence of Tukia's and Peltonen's definitions follows using Peltonen's computation of the $h_{i}$-s (and $\operatorname{diam} \sigma$ ), which is given - inter alia - in [71]. However, the proof requires additional notations and definitions as well as some quite extensive technical details. Therefore, we refer the reader to the original paper of Peltonen where the connection between $\varphi_{P}$ and $h$ (hence $\varphi, \varphi_{T}$ ) is given (even if not quite explicitly).

Munkres' definition is, for the specific case of Euclidean simplices ${ }^{21}$, somewhat of a compromise between Cheeger's and Tukia's ones:

$$
\varphi_{M}=\frac{\operatorname{dist}(b, \partial \sigma)}{\operatorname{diam} \sigma},
$$

where $b$ denotes the barycenter of $\sigma$ and $\partial \sigma$ represents the standard notation for the boundary of $\sigma$ (i.e the union of the $(n-1)$-dimensional faces of $\sigma$ ). From the considerations above (and from [65], Section 9) it follows that for the Euclidean case, this definition of thickness is also equivalent to the previous ones.

Remark 4.7. Fu [37] also introduces a definition of fatness that (up to the quite different notation) is identical to that of [29], when restricted to individual simplices. However, for triangulations, his definition exceeds, in

\footnotetext{
${ }^{21}$ But only in this case, as it represents a generalization of the Euclidean case for general simplices (as befitting Differential Topology goals).
} 
general, the one given in [29], due to the fact that Fu discards, in his use of fatness, the requirement that the approximations become arbitrarily fine (see also Remark 4.12 above).

Remark 4.8. Of course, there exists a (well know) duality between the spherical distances and dihedral angles, as expressed by the Gram determinant (see, e.g. [59] and the references therein), but unfortunately this duality does not hold precisely in the case that we are interested in, that is constant sectional curvature $K \equiv 0$, so we do not discuss it here. Let us note only the fact that this type of analysis involves a generalization of the Cayley-Menger determinant.

To complete the circle - so to say - we should emphasize that, given the dihedral angles and the areas of the $(n-1)$-faces of the Euclidean (piecewise flat) triangulation, one can calculate the edge lengths. For example, one can devise explicit computations for the case important in Ricci calculus, i.e. 4-simplices, minus a number of so called "dangerous configurations", (and implicit ones for the general case) in [33]. (A related formula - explicit for the 3-dimensional case - this time involving the Cayley-Menger determinant, can be found in [18], p. 344.)

4.1.1. Thickness, metric curvature and excess The connection between thickness and excess is quite obvious: A 2-simplex is thick iff it its excess is bounded away from 0 . Note, however, that one can not assert that higher dimensional simplices are thick iff all their 2-dimensional faces are. Indeed, one can easily construct, for example, 3-dimensional tetrahedra with regular base and the other faces congruent to each other and having the common vertex at $\varepsilon$ distance from the barycenter of the base.

We do not explore here the full depth of the interconnections between these three concepts (namely thickness, metric curvature and excess), and we postpone such analysis for further research except the case of principal curvatures which we shall investigate in some detail in the next section. However, we can not mention in this context the (by now classical) AbreschGromoll Theorem [2], that connects, in the Riemannian setting, between thickness (or rather "slimness" - it's very opposite), excess and Ricci curvature. (For a fitting version for $P L$ manifolds, see [95] as well as the sequel article [100].)

It is also important to underline the fact that, to obtain good approximations of curvatures of $P L$ manifolds, one needs, in fact, to start with triangulations having principal metric curvatures - see discussion in Section 4.4 below. (The other requirement is, of course, the metric approximation being arbitrarily fine, that is that the mesh of triangulation converges to zero.) 
Remark 4.9. The transparency, so to say, of the connection of Haantjes curvature with the notion of excess, thence to fatness, as detailed above, represents yet another reason why, for our geometric ends, we prefer it in lieu of the better known Menger curvature.

\subsection{Curvatures' approximation}

The approximation theory of Lipschitz-Killing curvatures (hence, by Remarks 3.64 and 3.66 of such basic quantities as volume, scalar curvature, mean (inward) curvature of the boundary) rests on the by now classical work of Cheeger et al. [29], the basic result therein being

Theorem 4.10 ([29]). Let $M^{n}$ be a compact Riemannian manifold, with or without boundary, and let $M_{i}^{n}$ be a sequence of fat PL (piecewise flat) manifolds, that are secant approximations of $M^{n}$, converging to $M^{n}$ in the Hausdorff metric. Denote by $\mathfrak{R}$ and $\mathfrak{R}_{i}$ respectively, the Lipschitz-Killing curvatures of $M^{n}, M_{i}^{n}$. Then $\mathfrak{R}_{i} \rightarrow \mathfrak{R}$ in the sense of measures.

Remark 4.11. 1. One can fatly triangulate the smooth manifold $M^{n}$ and obtain the desired approximation results for curvatures using directly and explicitly the intrinsic metric, not just $P L$ (Euclidean) approximations [77], [29] - see also Section 4.3 below, in particular Formulas (4.18), (4.19) and Remark 4.25.

2. As noted above, Theorem 4.10 is given essentially in terms of the intrinsic geometry of $M^{n}$. A similar characterization of the curvature measures in terms of the extrinsic geometry (of embeddings in $R^{n}$ ) is given in [37].

Remark 4.12. The condition that the triangulation necessarily becomes arbitrarily fine is, in fact, too strong if the manifold contains large flat regions. (A motivational example, widely noted and exploited in Computer Graphics, is that of a round cylinder in $\mathbb{R}^{3}$.) Also, we have noted in [84], [101] the need and possibility of a triangulation with variable density of vertices, adapting to curvature. (Recall also that in [37] the hypothesis in the definition of fatness requiring that the mesh of the triangulation converges to zero is discarded).

Remark 4.13. A similar result for the Einstein tensor, was obtained by Bernig - see [15] and the references within. Recall that, in the smooth case, the Einstein tensor is defined as

$$
E=\frac{\text { scal }}{2} g-\text { Ric. }
$$


The quest (indeed, the need) for fat triangulations as a prerequisite for Theorem 4.10, is hardly surprising, in view of the characterization of fat triangulations as being precisely those triangulations having dihedral angles bounded from below (Proposition 4.2) and in view of the following expression of the Lipschitz-Killing curvatures in terms of dihedral angles (see [29] for the proof):

$$
\begin{aligned}
R^{j}= & \sum_{\sigma^{n-j}}\left\{1-\chi\left(L\left(\sigma^{j}\right)\right)+\sum_{l} \measuredangle\left(\sigma^{n-j}, \sigma^{n-j+i_{1}}\right) \cdots \measuredangle\left(\sigma^{n-j+i_{1-1}}, \sigma^{n-j+i_{1}}\right) .\right. \\
& \left.\cdot\left[1-\chi\left(L\left(\sigma^{n-j+i_{l}}\right)\right)\right]\right\} \operatorname{Vol}\left(\sigma^{n-j}\right),
\end{aligned}
$$

where $L\left(\sigma^{j}\right)$ denotes the (spherical) link of $\sigma^{j}$, and $\measuredangle\left(\sigma^{i}, \sigma^{j}\right)$ is the internal dihedral angle of $\sigma^{i}<\sigma^{j} ; \measuredangle\left(\sigma^{i}, \sigma^{j}\right)=\operatorname{Vol}\left(L\left(\sigma^{i}, \sigma^{j}\right)\right.$, where the volume is normalized such that $\operatorname{Vol}\left(\mathbb{S}^{n}\right)=1$, for any $n$. (See [29] for further details.) (Here $\chi$, Vol denote, as usual, the Euler characteristic and volume of $\sigma^{k}$, respectively.)

We can further exploit (and enhance) Theorem 4.10 if provided with a results ensuring the existence of thick triangulations. One such result is

Theorem 4.14 ([81]). Let $M^{n}$ be a connected, oriented $n$-dimensional ( $n \geq$ 2) submanifold of $\mathbb{R}^{N}$ (for some $N$ sufficiently large), with boundary, having a finite number of compact boundary components, and such that one of the following condition holds:

(i) $M^{n}$ is of class $\mathcal{C}^{r}, 1 \leq r \leq \infty, n \geq 2$;

(ii) $M^{n}$ is a PL manifold and $n \leq 4$;

(iii) $M^{n}$ is a topological manifold and $n \leq 3$.

If the boundary components admit fat triangulations of fatness $\geq \varphi_{0}$, then there exist a global fat triangulation of $M^{n}$.

Remark 4.15. In fact, the conditions on the compactness and boundedness of the boundary components in the theorem above are too strong, as indicated by the results in [82], [84], where the theorem above was shown to hold also for (hyperbolic) manifolds with infinitely many boundary components (as well as for more general spaces). The role of the conditions in question is to exclude certain "pathological" cases.

Moreover, given the triangulation results of [65], [29] for manifolds without boundary, the following (important in the sequel) corollary follows immediately: 
Corollary 4.16 ([81]). Let $M^{n}$ be a Riemannian manifold satisfying the conditions in the statement of Theorem 4.14 above.

Then $M^{n}$ admits a fat triangulation.

Remark 4.17. For a similar earlier result see [25], as well as [7]. ${ }^{22}$

The immediate differential geometric consequence of Theorems 4.10 and 4.14, as well as Corollary 4.16 - where by "immediate" we mean here that it can be directly inferred by applying the methods of [29] - is the following:

Theorem 4.18. Let $N=N^{n-1}$ be a not necessarily connected manifold, such that $N=\partial M, M=M^{n}$, where $M^{n}$ is, topologically, as in the statement of Theorem 4.14.

(i) If $M, N$ are $P L$ manifolds, then the Lipschitz-Killing curvature measures of $N$ can be extended to those of $M$. More precisely, there exist Lipschitz-Killing curvature measures $\mathfrak{R}=\left\{R^{j}\right\}$ on $\bar{M}=M \cup N$, such that $\left.\mathfrak{R}\right|_{N}=\mathfrak{R}_{N}$ and $\left.\mathfrak{R}\right|_{M}=\mathfrak{R}_{M}$, except on a regular (arbitrarily small) neighbourhood of $N$, where $\mathfrak{R}_{N}, \mathfrak{R}_{M}$ denote the curvature measures of $N, M$ respectively.

(ii) If $M, N$ are smooth manifolds, then the same holds, but only in the sense of measures.

Remark 4.19. Here it is important to recall that $\left.R^{j}\right|_{\partial M^{n}}=H^{j}$ and, in the case of $P L$ manifolds, it represents the contribution of the $(n-j)$-dimensional simplices that belong to the boundary. (For an explicit formula, see any of the formulas (3.23), (3.38) or (3.39) of [29].)

Remark 4.20. In view of the previous Remark, Theorem 4.18 above can be considered, in a sense, as the "reverse" of the result of [29], Section 8, regarding the convergence of the boundary measures.

4.2.1. An alternative approach A different, quite natural, approach to the approximations of curvatures of smooth manifolds by the discrete curvature of $P L$ (piecewise flat) approximations stems from Formula 3.61. Here one regards the relevant edges of a fine enough triangulation both as the principal vectors (i.e. principal directions $\times K_{\min }$, respectively $k_{\text {Max }}$ ). Let us examine the feasibility of this method of approximating curvatures:

1. For $P L$ manifolds, this approach would be somewhat naive, if applied directly, as it gives only approximative results. (This is a consequence of the fine interplay between the necessity of ensuring the fatness of the

\footnotetext{
${ }^{22}$ The author is indebted to Marc Troyanov for bringing to his attention Attie's
} work [7]. 
triangulation, simultaneously with a good sampling of the direction in the tangential plane (or rather cone) - see [87].) One can overcome this obstacle by using an extension of the notion of principal curvatures to a far larger class of geometric objects than mere smooth or even PL manifolds (see, e.g. [121]), by passing to the so called generalized principal curvatures. Unfortunately, in order that we may be able to present even very succinctly this tool stemming from Federer's classical work [36], we have to introduce additional of technical definitions and notations.

Let $X$ be an arbitrary set in some $\mathbb{R}^{N}$ and let $X_{\varepsilon}$ denote the $\varepsilon$ neighbourhood of $X$. Then, for for small enough $\varepsilon>0, \partial X_{\varepsilon}$ is a $\mathcal{C}^{1,1}$-hypersurface. ${ }^{23}$ More precisely, $\varepsilon$ has to be strictly smaller than the reach of $X$,

$$
\operatorname{reach}(X)=\sup \left\{r>0 \mid \forall y \in X_{r}, \exists ! x \in X \text { nearest to } y\right\}
$$

Moreover, the reach itself has to be strictly positive.

It follows that $\partial X_{\varepsilon}$ admits principal curvatures (in the classical sense) $k_{i}^{\varepsilon}(x+\mathbf{n})$ at almost any point $p=x+\mathbf{n}$, where $\mathbf{n}$ denotes the normal unit vector (at $x$ ). Define the generalized principal curvatures by: $\kappa_{i}(\varepsilon, \mathbf{n})=\lim _{\varepsilon \rightarrow 0} k_{i}^{\varepsilon}(x+\mathbf{n})$. Then $\kappa_{i}(\varepsilon, \mathbf{n})$ exist $\mathcal{H}^{N-1}$-a.a. $(x, \mathbf{n})$. (Here $\mathcal{H}$ denotes again the Hausdorff measure.)

Using this generalization of principal curvatures, one can retrieve a proper analogue of Formula 3.61, namely

$$
\begin{aligned}
C_{j}(X, B)= & \int_{\operatorname{nor}(X)} \mathbf{1}_{B} \prod_{i=1}^{N-1} \frac{1}{\sqrt{1+\kappa_{i}(x, \mathbf{n})^{2}}} S_{N-1-j}\left(\kappa_{1}(x, \mathbf{n}), \cdots\right. \\
& \left.\cdots, \kappa_{N-1}(x, \mathbf{n})\right) d \mathcal{H}^{N-1}(x, \mathbf{n})
\end{aligned}
$$

where $C_{j}(X, B)$ denote the so called Lipschitz-Killing curvature measures (see [121] and the bibliography therein for details), $B$ being a bounded Borel set in $\mathbb{R}^{N}$, and nor $(X)$ denotes the (unit) normal bundle of $X$ :

$$
\operatorname{nor}(X)=\left\{(x, \mathbf{n}) \in \partial X \times \mathbb{S}^{N-1} \mid \mathbf{n} \in \operatorname{Nor}(X, x)\right\},
$$

${ }^{23}$ In general, $\partial X_{\varepsilon}$ is a $(N-1)$-dimensional manifold with Lipschitz outer unit normal field, for any $\varepsilon \in(0, \operatorname{reach}(X))$, where the definition of reach is given in $(4.14)$ - see [36]. 
where $\operatorname{Nor}(X, x)=\left\{\mathbf{n} \in S_{N-1} \mid\langle\mathbf{n}, v>\leq 0, v \in \operatorname{Tan}(X, x))\right\}$ is the normal cone (to $X$ at the point $x \in T$ ), dual to the tangent cone (to $X$ at the point $x \in T)$.

Using the convergence properties of the generalized principal curvatures and of the Lipschitz-Killing curvature measures (again, see [121] and the bibliography therein), the result now follows along the lines of the second part of our first proof of the theorem.

Remark 4.21. The idea of passing to a smooth (enough) surface close to the original given data set is a common one in Imaging and Graphics, even though the precise method presented above is not the standard one and appears, perhaps, to be rather technical. Moreover, this approach is similar in concept (even though based on a very different mathematical apparatus) to the one based on smoothings, approach that will be employed extensively in the sequel.

2. The case of smooth manifolds follows easily by following the same scheme as in the main approach in [29].

\subsection{A generalization: almost Riemannian manifolds}

Theorems 4.14 and 4.18 above admit generalizations (see also Remark 4.25 below), of which we bring here a rather direct one. We begin with the following definition (cf. [106]):

Definition 4.22. A metric space $(M, d)$ is called an almost Riemannian space iff

1. $M$ is a smooth manifold;

2. There exists a (smooth) Riemannian metric $g$ on $M$ and a constant $C_{0}>0$, such that, for any $x \in M$, there exists a neighbourhood $U(x)$, such that

$$
C_{0}^{-1} d(y, z) \leq \operatorname{dist}_{g}(y, z) \leq C_{0} d(y, z),
$$

for all $y, z \in U(x)$.

The basic example of an almost metric space (beyond the trivial one $d \equiv g$ ) is given by any smooth (Riemannian) manifold embedded in some $\mathbb{R}^{N}$ and $d$ be the Euclidean distance in $\mathbb{R}^{N}, d=$ dist $_{E u c l}$, i.e. precisely the setting which we are concerned: the secant approximation of an embedded smooth manifold, with its Euclidean (ambient) metric is a almost Riemannian manifold (relative, so to say, to the approximated smooth manifold). 
According to [106], this holds, in fact, for any $C_{0}>0$, albeit at the price of locality (i.e. the size of the neighbourhood $U(x)$ depends very much on the point $x$ ) and cannot be apriorily be asserted (and, in fact, on the large scale, the to distance (geometries) might - and usually do - differ quite widely). However, we can do a bit better, by requiring that the simplices of the approximations are fat. Even if this requires some "preprocessing" of the given manifold this can be done (this being the starting point of this paper). Then, for any triangulation patch, we have the following estimates:

1. If $M$ has no boundary, then, by [71]

$$
\frac{3}{4} d_{g}(y, z) \leq d_{E u c l}(y, z) \leq \frac{5}{3} d_{g}(y, z)
$$

2. If $M$ has boundary, then, by [81]

$$
\frac{3}{4} d_{g}(y, z)-f(\theta) \eta_{\partial} \leq d_{E u c l}(y, z) \leq \frac{5}{3} d_{g}(y, z)+f(\theta) \eta_{\partial M}
$$

where $f(\theta)$ is a constant depending on the $\theta=\min \left\{\theta_{\partial M}, \theta_{\text {int } M}\right\}-$ the fatness of the triangulation of $\partial M$ and int $\mathrm{M}$, respectively, and $\eta_{\partial}$ denotes the mesh of the triangulation

Even though in the general case we can not produce estimates as precise as (4.18) and (4.19) above, (4.17) still holds. Since $M^{n}$ is a topological manifolds, we can triangulate it (using Munkres' classical results) and, furthermore, we can pass from $d(y, z)$ to dist $_{g}$ and back in a controlled manner, using (4.17), thus allowing us to apply the fattening techniques of [29]. ${ }^{24} \mathrm{It}$ follows that Theorems 4.14 generalizes to almost Riemannian manifolds, and we formalize this observation as

Theorem 4.23. Let $(M, d)$ be an almost Riemannian manifold, where $M$ satisfies the conditions in the statement of Theorem 4.14. Then it admits a fat triangulation.

Remark 4.24. Of course, one would also like to obtain a version of Theorem 4.18 adapted to almost Riemannian manifolds. However, this eludes us so far, since even a proper definition of the Lipschitz-Killing curvatures of an almost Riemannian manifold is not quite evident.

\footnotetext{
${ }^{24}$ The $\varepsilon$-moves employed in [29] show that only the metric properties suffice to ensure fatness. (Recall also the discussion in Section 4 above.)
} 
Remark 4.25. Besides the slight generalization (and its applications) presented above, there exist other, perhaps more less immediate ones - see [84], [86]. In particular, we noted the existence of fat triangulations of Lipschitz manifolds. Since Alexandrov spaces are, by results of Perelman [72] and Otsu and Shioya [68], Lipschitz manifolds a.e., fat triangulations of such objects, and their differential geometric implications are certainly worth to explore, especially the role of Alexandrov spaces in Graphics, Imaging, etc., and also in Regge calculus - see Section 4.4.1 below and also [93], [93].

Note also that Semmes stresses that the condition in the definition of almost Riemannian manifolds, of $M$ being a topological manifold is far too strong. We can still use Semmes' original Definition 4.22 for $P L$ (or polyhedral) manifolds (at least in dimension $n \leq 4$ ) by considering smoothings (see [65]) and also considering, instead of the original $P L$ (polyhedral) metric $g$, its smoothing $\tilde{g}$ - see [93], [93] for details and another application of this technique. (In fact, any topological manifold of dimension $n \leq 3$, admits a $P L$ structure - see, e.g., [65] - therefore one can construct almost Riemannian manifolds of dimension 2 and 3 , by starting with a topological manifold and considering, for example, the combinatorial metric on a compatible $P L$ structure, etc.)

Remark 4.26. While, as we have noted above, not all the differential geometric consequences of the existence of fat triangulations of almost Riemannian manifolds are, at this point in time, accessible to us, this generalization is not gratuitous, either. Indeed, the fact that $P L$ manifolds - satisfying certain additional technical conditions (see [106]), that we do not bring here in order not to deviate to much from the main ideas - are almost Riemannian manifolds, shows that they also admit differential forms satisfying additional properties - see [106], Theorems 17.3 and 17.10. This differential forms are related to the construction of certain Lipschitz mappings that are connected, in their turn, to the existence of sufficiently large families of curves connecting two given points, with lengths not deviating too much from the distance between the two points [106]. We shall investigate elsewhere the implications (and relevance) of the existence of such curves for $P L$ manifolds [91]. However, we formalize the fact above as:

Proposition 4.27. Let $(M, d)$ be a PL manifold. Then there exists on $M$ bounded measurable (resp. locally integrable) differential forms that satisfy the conditions in [106], Theorem 17.3 (resp. [106], Theorem 1.10).

Moreover, given the following facts: (a) If $\left\{P_{m}\right\}_{m \in \mathbb{N}}$ is a sequence of $n$ dimensional polyhedral manifolds, converging, in secant approximation to $M^{n} \subset \mathbb{R}^{N}$, then the convergence is also in the Lipschitz sense (see [37], 
Section 3); (b) Theorems Theorem 17.3 and 1.10 of [106] are proved using solely properties of Lipschitz functions (with values in $\mathbb{S}^{n}$ ), we formulate the following conjecture, whose full proof we shall bring elsewhere [92]:

Conjecture 1. Let $M^{n} \subset \mathbb{R}^{N}$ be a $C^{1,1}$ compact manifold with boundary, and let $\left\{P_{m}\right\}_{m \in \mathbb{N}}$ be a sequence of fat $P L$ (polyhedral) manifolds with boundary closely inscribed ${ }^{25}$ in $M^{n}$, Hausdorff converging to $M^{n}$. Denote by $\theta_{0, m}, \theta_{T, m}$, $\Lambda_{1, m}$ and $\Lambda_{2, m}$ the bounded measurable, respectively locally integrable, differential forms given by [106], Theorems 17.3 and 1.10, respectively. Then $\theta_{0, m} \rightarrow \theta_{0}, \theta_{T, m} \rightarrow \theta_{T}, \Lambda_{1, m} \rightarrow \Lambda_{1}, \Lambda_{2, m} \rightarrow \Lambda_{2}$, where $\theta_{0}, \theta_{T}, \Lambda_{1}, \Lambda_{2}$ are bounded measurable, respectively locally integrable differential forms on $M^{n}$, satisfying the conditions in [106], Theorems 17.3 and 1.10, respectively. Here the convergence should be understood in the sense that it occurs only where it makes sense, that is on the space of forms on the common set of $\left\{P_{m}\right\}_{m \in \mathbb{N}}$ and $M^{n}$, i.e. on the vertices of the polyhedral manifolds $P_{m}$.

As we have seen above, there are quite strong geometric facts than can be asserted about almost Riemannian manifolds.

\subsection{Applications}

We investigate the applications of Theorem 4.18 to (a) the Regge calculus, and (b) Computer Graphics and related fields.

4.4.1. Regge calculus Looking for the applications of the results above in the context of Regge calculus [77], is only natural, given that the original motivation of [29] (and its precursory, more Physics oriented, [28]) stemmed precisely from therein. Indeed, as emphasized in [29], Gauss (scalar) curvature $K \equiv R^{2}$ and mean curvature $H \equiv H^{2}$ are relevant to the Hilbert action principle, thence to the derivation of the Einstein field equations.

4.4.1.1. An immediate consequence As we have already noted above, our result represents a "reverse version" of a result of [29]. Its relevance to (Discrete) General Relativity is accentuated by contemporary works such as [107]. Indeed, our main result 4.18 has as a (rather simple) consequence the possibility of extending the intrinsic the intrinsic differential structure from the surface singularity to its interior, that is, starting from a horizon of prescribed (and arbitrary) geometry, constructing an asymptotically flat structure. This is possible not only for the immediate (rather trivial) case

${ }^{25}$ See [37], p. 179 for the precise definitions. 
of smooth singularities, e.g. Wheeler wormholes [119] (i.e. such that $S \simeq \mathbb{S}^{2}$, where $S \subset \partial M^{3}$ ) that constituted an important motivational phenomenon in Regge's original paper [77], but also for the more interesting case of Wheeler Foam [120], i.e. non-smooth singularities (for which topology and curvature change with scale) ${ }^{26}$

4.4.1.2. Purely metric Regge calculus The Physical motivation of the study of the curvatures convergence problem raises a few natural, interrelated, questions:

Question 1. The PL spaces are still not the discrete metric spaces (lattices) sought for in quantum field theory. Can one discard this restriction?

The answer turns out to be affirmative, as we indicate below, if one discards the angular defect approach in favor of working with metric curvatures

Question 2. Since Regge's drive was to find a purely metric (discrete) formulation of Gravity, the presence of angles in the Lipschitz-Killing curvatures is a bit "unesthetic", as already stressed above. Hence: can one (nontrivially) formulate Theorem 4.18 (and its consequences) solely in metric terms?

Again, the answer to this question, as for the previous one, is positive. Our suggested approach to its solution is, evidently, to make appeal yet once again to formula (3.61) to express the curvature at a vertex by principal curvatures (which is easy - and natural - to determine for a piecewiseflat surface). Use then results of [18], Section 10 (see also Section 3.1.2) above, to show that for piecewise-flat manifolds, using metric curvatures (Menger and/or Haantjes) one can approximate well (in fact: as well as desired) smooth (classical) curvature. In consequence one can compute the Gauss and mean curvatures of piecewise-flat surfaces, with a clear and well established importance in Graphics, etc. (See also the discussion in the next section.)

However, these are only approximations with limited convergence properties - see [97], [80] for the metric curvatures aspect and also [112], [32], amongst others, for the more general problem of approximating principal curvatures. The problem is that, in order to ensure convergence for the

\footnotetext{
${ }^{26} \mathrm{An}$ amusing (almost "Sci-Fi") consequence of this result is the fact that it allows for a continuous "gluing" of the geometry of the given "Universe", across the black-hole singularity (i.e. the common boundary), to the "Alternate Universe", without any distortion (hence without observable changes for an observer), except on a arbitrarily small, symmetric tubular neighbourhood of the singularity.
} 
Cheeger et al. process, the triangulation has not only to converge in mesh to 0 , it also has to remain fat. However, to ensure a "good sampling" of the directions on a surface (so to ensure good approximation of the principal curvatures), one necessarily has to produce samplings whose edges directions are arbitrarily dense in the tangent plane (cone) at a given point (vertex), in contradiction with the previous fatness constraint. (Note, however, that this problem does not exist if one is willing to be content with approximate results, correct up to a predetermined error. One should also keep in mind Remarks 4.12 and 4.7 regarding Fu's work, as being pertinent to this issue.)

Moreover, given the fact (discussed above), that one can not increase as desired the number of triangles adjacent to a vertex, there exist only a very limited number of directions to "choose" from. Thus one is quite restricted in adding new directions (thus to better "sample" the surface, so to say) without negatively affecting the fatness of the triangulation. ${ }^{27}$

How to "get rid" of this problem?

- "Mix" the angles in the manner described in detail in [82], to obtain angles whose measure is close to their mean (i.e. $\pi / \operatorname{deg}(v)$ - where $\operatorname{deg}(v)$ denotes the number of triangles adjacent to the vertex $v$. (This "trick" works if a smoothness condition (albeit, minimal) is imposed on the manifold - it certainly holds for triangulated surfaces.)

- Add directions by considering the PL-quasi-geodesic and "normalize" (by projection on the normal plane) - see [98], [97] and the references therein for details and some numerical results.

- The approximation of principal curvatures being, as already noted above, notoriously difficult - see [64] [112], [76], [32] - one seeks other, perhaps less direct strategies. Such a method does, in indeed exist, embodied by the generalized principal curvatures, as we have detailed above. Passing to smooth surfaces allows for the use of a wide scale of well developed and finely honed methods of Graphics and related fields. Furthermore, it clearly compensates for its departure from the given (data) set of discrete/geometric $P L$ object by its generality whence its applicative potential in a very general setting.

As we have seen above, we can compute the principal curvatures of $S$ via those of the smoother surface $S_{\varepsilon}$, at least up to some infinitesimal distortion. However, to determine the full curvature tensor, in the case of higher dimensional manifolds, suffices to determine the Gauss curvature of

\footnotetext{
${ }^{27}$ In practice (Graphics, etc.) even angles $\pi / 12$ are already problematically small!...
} 
all the 2-sections (see observation above). So, for the full reconstruction of the curvature tensor it is not necessary to determine the principal curvatures, suffices to find the sectional curvatures. We shall show shortly how we this can be done.

Remark 4.28. It is natural to ask the question whether it is possible to compute the Lipschitz-Killing curvatures, starting from the defining Formula (3.59), that is if one can compute the necessary curvature 2-forms and connection 1-forms. The answer seems to be positive, even though, till recently this was only a mainly theoretical possibility (see [110]). However, after the appearance of the computational exterior differential calculus, introduced by $\mathrm{Gu}$ [47] and $\mathrm{Gu}$ and Yau [49], and embraced and developed since then by many others, this approach appears quite feasible, at least in dimensions 2 and 3.

Another related question is whether it is possible to determine other important curvatures (e.g. Ricci and scalar) as well as the Lipschitz-Killing curvatures, via the sectional curvatures, without appeal to principal curvatures (and Formula (3.61) or $\left.(4.15)^{28}\right)$. We have tackled this problem in [48] and presented a partial, possible solution and some of its consequences (see also [100]).

4.4.2. Computer graphics Polygonal/polyhedral (mainly triangular/ tetrahedral) meshes are the basic representations of geometry, employed in a plethora of related fields, such as Computer Vision, Image Processing, Computer Graphics, Geometric Modeling and Manufacturing. Curvature analysis of this type of data sets plays a major role in a variety of applications, such as reconstruction, segmentation and recognition and non photorealistic rendering (see the bibliography included in [61] for some of the vast - and ever developing - literature on the subject).

It is perhaps needles to mention that many of the facts and techniques discussed with regard to the Regge calculus are relevant in this context as well. Amongst these we mention $P L$ geodesics and Haantjes curvature for the determination principal curvatures (see [61] for a discussion and [97] for an implementation and some numerical results), "mixing" of the angles and, certainly not the least, the $P L$ metric Ricci curvature and flow.

Moreover, since, as already mentioned above, $R^{0} \equiv$ volume, $R^{2} \equiv$ scalar curvatureand $H^{1} \equiv$ area boundary, $H^{2} \equiv$ mean curvature for inward normal, etc., there exists a relationship between the main subject of this paper and the fields mentioned above. Indeed, one such connection was already

\footnotetext{
${ }^{28}$ or, (4.13), for that matter.
} 
mentioned in Remark 4.20 above. However the connection is deeper and less trivial than this. Indeed, in Computer Graphics, Computer Aided Geometric Design, etc. it has become customary lately to compute so called volumetric curvatures (see, e.g. [108]), and Graphics (see [50] and the bibliography therein). This amounts, in fact, to the computation of the curvatures (Gauss and mean) of surfaces evolving in time. We take this opportunity to note that, while surely this approach has its merit and applications, a proper "volumetric", i.e. 3-dimensional curvature (measures), would entail the computation of a namely sectional, scalar and Ricci curvatures. In all fairness, we should add that, for Computer Graphics, where usually the data is already embedded in $\mathbb{R}^{3}$, thus endowed with the Euclidean (flat) geometry of the ambient space, such computations are, therefore, rather meaningless.

Clearly, the metric approach to the computation of $P L$ (and polyhedral) manifolds considered above is more relevant in practice for the applicative fields considered above. This is particularly true for 2-dimensional manifolds, with further emphasis on the types of surface (square grids) traditionally employed in Imaging, since, as we have already noted, the notion of Ricci curvature for the dual complex and the one for the original (given) complex, have the same geometric significance and even coincide, perhaps up to a constant.

We conclude this section by noting that estimation of curvatures, mainly of mean curvature, is also important (mostly via the Cahn-Hilliard equation) in physically motivated applications - see, e.g. [53], amongst many others.

Remark 4.29. We can not conclude this section without pointing out that the reverse connection between curvature and thickness of a triangulation is well established. More precisely, to construct a thick triangulation of a manifold, one chooses its vertices according to a (metric) sampling density given by its curvature. This is, by now, a subject explored (and exploited) in detail - see [71], [81], [85], [104], [88], [86], [101], [102].

\section{Conclusion and future work}

We have introduced the various metric curvatures, both for curves and surfaces and discussed their extensions and applications to the definition of metric curvature for $P L$ (and polyhedral) spaces, and we investigated the relationship with - by now the well established in Graphics, Imaging and Manifold Learning - notion of Gromov-Hausdorff convergence. We next presented a number of applications, especially in Imaging (e.g. via a metric curvature for wavelets), but also for networks. In addition, we sketched a 
number of other possible applications, mainly to Graphics and Regge Calculus, via metric versions of the Lipschitz-Killing curvatures, and proposed a few more possible, related directions of study.

To be sure, there are still many details to be filled in and concrete implementations to be made. Amongst these, we mention a possible "metric Nash" embedding result with multiple applications in Complex Networks, as well as an implementation of the metric Lipschitz-Killing in Graphics and Manifold Learning and Regge Calculus. In this last application, of particular interest would be a comparison with the simplicial Regge calculus of Alsing et al. Amongst the most natural - and important - directions of further study that present themselves, are the development of fitting Ricci flows for the Wald curvature, with applications to Network Theory and practice, and for piecewise flat manifolds, aimed for applications in Regge Calculus. In this last instance, a problem that imposes itself, is the comparison of the metric (Wald) flow and Alsing's simplicial one.

We hope that the classical notions and results, their applications so far in practice, as well as open problems, will present themselves a an interesting, attractive and worthwhile pursuing field of study for other scientist in the various relevant fields.

\section{Acknowledgements}

The author would like to thank his former students who helped produce some of the figures in the text; to Jürgen Jost for his warm hosting at the "Max Planck" Institute, Leipzig; to Manor Mendel whose support meant so much; to Pascal Romon and Laurent Najman who's advice assisted in cleaning the text and making it more clear; and last - but certainly not least! - to David Xianfeng $\mathrm{Gu}$, whose keen interest and warm encouragement made this project possible.

\section{References}

[1] I. Abraham, Y. Bartal and O. Neiman, Advances in Metric Embedding Theory, Advances in Mathematics, 228(6), 3026-3126, 2011. MR2844939

[2] U. Abresch and D. Gromoll, On complete manifolds with nonnegative Ricci curvature, J. Amer. Math. Soc., 3(2), 355-374, 1990. MR1030656

[3] S. B. Agard and F. W. Gehring, Angles and Quasiconformal Mappings, Proc. London Math. Soc., 3(1), 1-21, 1965). MR0178140 
[4] S. B. Alexander and R. L. Bishop, Comparison theorems for curves of bounded geodesic curvature in metric spaces of curvature bounded above, Differential Geometry and its Applications, 6, 67-86, 1996. MR1384880

[5] P. M. Alsing, J. R. McDonald and W. A. Miller, The simplicial Ricci tensor, Class. Quantum Grav., 28, 155007 (17 pp), 2011. MR2822636

[6] E. Appleboim, Y. Hyams, S. Krakovski, C. Sageev and E. Saucan, The Scale-Curvature Connection and its Application to Texture Segmentation, Theory and Applications of Mathematics \& Computer Science, 3(1), 38-54, 2013.

[7] O. Attie, Quasi-isometry classification of some manifolds of bounded geometry, Math. Z. 216, 501-527, 1994. MR1288043

[8] T. A. Banchoff, Critical points and curvature for embedded polyhedra, J. Differential Geometry, 1, 257-268, 1967. MR0225327

[9] T. A. Banchoff, Critical Points and Curvature for Embedded Polyhedral Surfaces, Amer. Math. Monthly, bf 77, 475-485, 1970. MR0259812

[10] B. Bahr and B. Dittrich, Regge calculus from a new angle, New Journal of Physics 12, (doi:10.1088/1367-2630/12/3/033010), 2010. MR2606604

[11] V. N. Berestovskii, Spaces with bounded curvature and distance geometry, Siberian Math. J. 16, 8-19, 1986. MR0847410

[12] M. Berger, Encounter with a Geometer, Part II, Notices of the AMS 47(3), 326-340, 2000. MR1740389

[13] M. Berger, A Panoramic View of Riemannian Geometry, SpringerVerlag, Berlin, 2003. MR2002701

[14] A. Bernig, Scalar curvature of definable Alexandrov spaces, Adv. Geom. 2, 29-55, 2002. MR1880000

[15] A. Bernig, Curvature bounds of subanalytic spaces, preprint, 2003.

[16] J. Bertrand, M. Diguet and V. Puiseux, Démonstration d'un théorème de Gauss, J. de Mathématiques, 13, 80-90, 1848.

[17] L. M. Blumenthal, Distance Geometry - Theory and Applications, Claredon Press, Oxford, 1953. MR0054981

[18] L. M. Blumenthal and K. Menger, Studies in Geometry, Freeman and Co., San Francisco, 1970. MR0273492 
[19] J. Bourgain, On Lipschitz embedding of finite metric spaces in Hilbert space, Israel J. Math, 46-52, 1985. MR0815600

[20] A. M. Bronstein, M. M. Bronstein, A. M. Bruckstein and R. Kimmel, Paretian Similarity for Partial Comparison of Non-rigid Objects, LNCS, 4485, 264-275, 2007.

[21] A. M. Bronstein, M. M. Bronstein and R. Kimmel, On isometric embedding of facial surfaces into S3, Proc. Intl. Conf. on Scale Space and PDE Methods in Computer Vision, 622-631, 2005.

[22] A. M. Bronstein, M. M. Bronstein and R. Kimmel, Three-dimensional face recognition, International Journal of Computer Vision, 64(1), 530, 2005.

[23] R. Brooks, Differential Geometry, Course Notes, Technion, 2003.

[24] D. Burago, Y. Burago and S. Ivanov, Course in Metric Geometry, GSM 33, AMS, Providence, 2000.

[25] S. S. Cairns, On the triangulation of regular loci, Ann. of Math. 35, 579-587, 1934. MR1503181

[26] M. Cassorla, Approximating compact inner metric spaces by surfaces, Indiana Univ. Math. J. 41, 505-513, 1992. MR1183356

[27] A. Cayley, On a Theorem in the Geometry of Position, Camb. Math. Jour., t. II, 267-271, 1941.

[28] J. Cheeger, W. Müller and R. Schrader, Lattice gravity or Riemannian structure on piecewise linear spaces, in Breitenlohner, P., and Dürr, H.P., eds., Unified theories of elementary particles, Lecture Notes in Physics 160, 176-188, Springer, Berlin, 1982. MR0822799

[29] J. Cheeger, W. Müller, and R. Schrader, On the Curvature of Piecewise Flat Spaces, Comm. Math. Phys. 92, 405-454, 1984. MR0734226

[30] H. S. M. Coxeter, Introduction to Geometry, 2nd ed., John Wiley \& Sons, 1969. MR0346644

[31] M. P. do Carmo, Differential Geometry of Curves and Surfaces, Prentice-Hall, Englewood Cliffs, N.J., 1976. MR0394451

[32] J. Dai, W. Luo, M. Jin, W. Zeng, Y. He, S.-T. Yau and X. Gu, Geometric accuracy analysis for discrete surface approximation, Computer Aided Geometric Design 24, 323-338, 2007. MR2339977

[33] B. Dittrich and S. Speziale, Area angle variables for general relativity, New Journal of Physics, 10(8), 083006, 2008. 
[34] M. P. do Carmo, Riemannian Geometry, Mathematics: Theory \& Applications, Birkhäuser, Boston, Mass., 1992. MR1138207

[35] H. Edelsbrunner, Geometry and topology for mesh generation, Cambridge University Press, Canbridge, 2001. MR1833977

[36] H. Federer, Curvature measures, Trans. Amer. Math. Soc. 93, 418-491, 1959. MR0110078

[37] J. H. G. Fu, Convergence of Curvatures in Secant Approximation, J. Differential Geometry, 37, 177-190, 1993. MR1198604

[38] Gehring, W. F. and Väisälä, J. The coefficients of quasiconformality, Acta Math. 114, pp. 1-70, 1965. MR0180674

[39] G. Gilboa, E. Appleboim, E. Saucan and Y. Y. Zeevi, On the Role of Non-local Menger Curvature in Image Processing, Proceedings of ICIP 2015, 2015.

[40] A. Gray, Tubes, Addison-Wesley, Redwood City, Ca., 1990. MR1044996

[41] A. Gray and L. Vanhecke, Riemannian geometry as determined by the volume of small geodesic balls, Acta Mathematica, 142, 157-198, 1979. MR0521460

[42] M. Gromov, Sign and geometric meaning of curvature, Rend. Sem. Mat. Fis. Milano, 61, 9-123, 1991. MR1297501

[43] M. Gromov, Metric structures for Riemannian and non-Riemannian spaces, Progress in Mathematics, 152, Birkhauser, Boston, 1999. MR1699320

[44] K. Grove and S. Markvorsen, Curvature, Triameter and Beyond, Bull. American Math. Soc. 27, 261-265, 1992. MR1152160

[45] K. Grove and S. Markvorsen, New extremal problems for the Riemannian recognition program via Alexandrov geometry, J. American Math. Soc. 8, 1-28, 1995. MR1276824

[46] K. Grove and P. Petersen, Bounding homotopy types by geometry. Ann. of Math. 128 (1988), 195-206. MR0951512

[47] X. Gu, Parametrization for surfaces with arbitrary topologies, PhD Thesis, Computer Science, Harvard University, 2002.

[48] D. X. Gu and E. Saucan, Metric Ricci curvature for PL manifolds, Geometry, vol. 2013, Article ID 694169, 12 pages, 2013. doi:10.1155/2013/694169. 
[49] X. Gu and S.-T. Yau, Computing Conformal Structures of Surfaces, Communications in Information and Systems, 2(2), 121-146, 2002. MR1958012

[50] X. D. Gu and S.-T. Yau, Computational Conformal Geometry, Advanced Lectures in Mathematics 3, International Press, Somerville, MA, 2008. MR2439718

[51] J. Haantjes, Distance geometry. Curvature in abstract metric spaces, Proc. Kon. Ned. Akad. v. Wetenseh., Amsterdam 50, 496-508, 1947. MR0021325

[52] J. Haantjes, A characteristic local property of geodesics in certain spaces, Proc. Kon. Ned. Akad. v. Wetenseh., Amsterdam 54, 66-73, 1951. MR0040675

[53] T. Haxhimali, A. Karma, F. Gonzales and M. Rappaz, Orientation selection in dendritic evolution, Nature Materials 5, 660-664, 2006.

[54] E. Heintze and H. Karcher, A general comparison theorem with applications to volume estimates for submanifolds, Ann. Scient. Ec. Norm Sup., 11, 451-470, 1978. MR0533065

[55] D. Hilbert, and S. Cohn-Vossen, Geometry and the Imagination, Chelsea, Providence, RI, 1952. MR0046650

[56] J. F. Hudson, Piecewise Linear Topology, Math. Lect. Notes Series, Benjamin, N.Y., 1969. MR0248844

[57] D. C. Kay, Arc curvature in metric spaces, Geometriae Dedicata 9(1), 91-105, 1980. MR0566441

[58] W. Klingenberg, A course in differential geometry, GTM 51, Springer, Berlin, 1974. MR0474045

[59] S. L. Kokkendorff, Polar duality and the generalized Law of Sines, J. Geom. 86, 140-149, 2006. MR2316927

[60] R. Krauthgamer, N. Linial and A. Magen Metric Embeddings - Beyond one-dimensional distortion, Discrete and Computational Geometry, 31, 339-356, 2004. MR2036942

[61] R. Lev, E. Saucan and G. Elber, Curvature Estimation over Smooth Polygonal Meshes using The Half Tube Formula, LNCS, 4647, 275289, Springer-Verlag, 2007. 
[62] N. Linial, E. London and Yu. Rabinovich, The geometry of graphs and some of its algorithmic applications, Combinatorica, 15, 215-245, 1995. MR1337355

[63] S. H. Lui, An Interview with Vladimir Arnol'd, Notices of the AMS, 44(4), 432-438, 1997. MR1435453

[64] R. Martin, Estimation of principal curvatures from range data, International Journal of Shape Modeling, 4, 99-111, 1998.

[65] J. R. Munkres, Elementary Differential Topology, (rev. ed.), Princeton University Press, Princeton, NJ, 1966. MR0198479

[66] J. Nash, The embedding problem for Riemannian manifolds, Ann. of Math. (2) 63, 20-63, 1956. MR0075639

[67] Y. Otsu, On manifolds with small excess, Amer. J. Math. 115, 12291280, 1993. MR1254733

[68] Y. Otsu and T. Shioya The Riemannian structure of Alexandrov spaces J. Differential Geom. 39(3) (1994), 629-658. MR1274133

[69] H. Pajot, Analytic Capacity, Rectificabilility, Menger Curvature and the Cauchy Integral, LNM 1799, Springer-Verlag, Berlin, 2002. MR1952175

[70] C. Pauc, Courbure dans les espaces métriques, Atti Acad. di Lincei, Serie 6 24, 109-115, 1936.

[71] K. Peltonen, On the existence of quasiregular mappings, Ann. Acad. Sci. Fenn., Series I Math., Dissertationes, 1992. MR1165363

[72] G. Perelman, Alexandrov's spaces with curvature bounded from below II, preprint, 1991.

[73] P. Petersen, Riemannian Geometry, Springer-Verlag, New York, 1998. MR1480173

[74] C. Plaut, Metric Spaces of Curvature $\geq k$, Handbook of Geometric Topology (Daverman, R. J. and Sher, R. B., editors), 819-898, Elsevier, Amsterdam, 2002. MR1886682

[75] G. Polya, An elementary analogue of the Gauss-Bonnet theorem, Amer. Math. Monthly, 61, 601-603, 1954. MR0066668

[76] H. Pottmann, J. Wallner, Y.-L. Yang, Y.-K. Lai and S.-M. Hu, Principal curvatures from the integral invariant viewpoint, Computer Aided Geometric Design, 24, 428-442, 2007. MR2359761 
[77] T. Regge, General relativity without coordinates, Nuovo Cimento, 19, 558-571, 1961. MR0127372

[78] T. Regge and R. M. Williams, Discrete structures in gravity, J. Math. Phys. 41, 3964, 2000. MR1768645

[79] C.V. Robinson, A Simple Way of Computing the Gauss Curvature of a Surface, Reports of a Mathematical Colloquium, Second Series, 5-6, 16-24, 1944. MR0011782

[80] E. Saucan, Surface triangulation - the metric approach, preprint (arxiv:cs.GR/0401023), 2004.

[81] E. Saucan, Note on a Theorem of Munkres, Mediterranean Journal of Mathematics, 2(2), 215-229, 2005. MR2184196

[82] E. Saucan, The Existence of Quasimeromorphic Mappings in Dimension 3. Conform. Geom. Dyn., 10, 21-40, 2006. MR2206314

[83] E. Saucan, Curvature - Smooth, Piecewise-Linear and Metric, in "What is Geometry?", Advanced Studies in Mathematics and Logic, 237-268, Polimetrica, Milano, 2006.

[84] E. Saucan, Remarks on the Existence of Quasimeromorphic Mappings, Cont. Math., 455, 325-331, 2008. MR2408179

[85] E. Saucan, Intrinsic Differential Geometry and the Existence of Quasimeromorphic Mappings, Revue Roumaine de Math. Pures et Appl., 54, 5-6, 565-574, 2009. MR2603792

[86] E. Saucan, Curvature based triangulation of metric measure spaces, Contemporary Mathematics, 554, 207-227, 2011. MR2884404

[87] E. Saucan, Fat Triangulations and Differential Geometry, preprint, (arXiv:1108.3529v1 [math.DG]), 2011.

[88] E. Saucan, Geometric Sampling of Infinite Dimensional Signals, Sampl. Theory Signal Image Process., 10(1-2), 59-76, 2011. MR2868033

[89] E. Saucan, Isometric Embeddings in Imaging and Vision: Facts and Fiction, Journal of Mathematical Imaging and Vision, 43(2), 143-155, 2012. MR2910879

[90] E. Saucan, On a construction of Burago and Zalgaller, The Asian Journal of Mathematics, 16(4), 587-606, 2012. MR3004279

[91] E. Saucan, Discrete Geodesics on Weighted Surfaces, in preparation, 2013. 
[92] E. Saucan, in preparation, 2013.

[93] E. Saucan, A Metric Ricci Flow for Surfaces and Its Applications, Geometry, Imaging and Computing, 1(2), 259-301, 2014. MR3396624

[94] E. Saucan, Metric Curvatures Revisited - A Brief Overview, bookchapter, to appear in Springer LNM.

[95] E. Saucan, Triangulated Surfaces as Alexandrov Surfaces I-Geometric Aspects, in preparation.

[96] E. Saucan and E. Appleboim, Curvature Based Clustering for DNA Microarray Data Analysis, LNCS, 3523, 405-412, Springer-Verlag, 2005.

[97] E. Saucan and E. Appleboim, Metric Methods in Surface Triangulation, Lecture Notes in Computer Science, 5654, 335-355, Springer, 2009.

[98] E. Saucan, E. Appleboim, E. Barak-Shimron, R. Lev and Y. Y. Zeevi, Local versus Global in Quasiconformal Mapping for Medical Imaging, J. Math. Imaging Vis., 32(3), 293-311, 2008. MR2442980

[99] E. Saucan, On a construction of Burago and Zalgaller, The Asian Journal of Mathematics, 16(4), 587-606, 2012. MR3004279

[100] E. Saucan, Metric Curvatures and their Applications II: Metric Ricci Curvature and Flow, in preparation, 2015.

[101] E. Saucan, E. Appleboim and Y. Y. Zeevi, Sampling and Reconstruction of Surfaces and Higher Dimensional Manifolds Journal of Mathematical Imaging and Vision, 30(1), 105-123, 2008. MR2384944

[102] E. Saucan, E. Appleboim and Y. Y. Zeevi, Geometric Approach to Sampling and Communication, Sampl. Theory Signal Image Process. 11(1), 1-24, 2012. MR3284395

[103] E. Saucan, C. Sagiv and E. Appleboim, Geometric Wavelets for Image Processing: Metric Curvature of Wavelets, Proceedings of SampTA 2009, http://hal.archives-ouvertes.fr/docs/00/49/54/56/PDF/ SampTAProceedings.pdf, 85-89.

[104] E. Saucan and M. Katchalski, The existence of thick triangulations an "elementary" proof, The Open Mathematics Journal, 2, 8-11, 2009. MR2529766

[105] E. Saucan and M. Katchalski, Fat Triangulations, Curvature and Quasiconformal Mappings, Axioms, 1(2), 99-110, 2012. 
[106] S. Semmes Finding curves on general spaces through quantitative topology, with applications to Sobolev and Poincaré inequalities, Selecta Mathematica, New Series, 2(2), 155-295, 1996. MR1414889

[107] B. Smith, Black hole initial data with a horizon of prescribed intrinsic geometry, Gen. Relativ. Gravit. (2009) 41:1013-1024 MR2506543

[108] O. Soldea, G. Elber and E. Rivlin, Global Segmentation and Curvature Analysis of Volumetric Data Sets Using Trivariate B-spline Functions, IEEE Transactions on Pattern Analysis and Machine Intelligence, 28(2), 265-278, 2006.

[109] Sommerville, D.M.Y.: An Introduction to the Geometry of N Dimensions, Dover Publications, N.Y., 1958. MR0100239

[110] R. Sorkin, The electromagnetic field on a simplicial net, J. Math. Phys., 16(12), 2432-2440, 1975. MR0389139

[111] M. Spivak, A Comprehensive Introduction to Differential Geometry, Vol. 2, Publish or Perish, Houston, TX, 1970.

[112] T. Surazhsky, E. Magid, O. Soldea, G. Elber and E. Rivlin, A Comparison of Gaussian and Mean Curvatures Estimation Methods on Triangular Meshes, Proceedings of the IEEE International Conference on Robotics and Automation Taipei, Taiwan 2003, 1021-1026, 2003.

[113] P. M. Topping, Lectures on the Ricci Flow, London Mathematical Society Lecture Note Series 325, Cambridge University Press, Canbridge, 2006. MR2265040

[114] T. Toro, Surfaces with generalized second fundamental form in $L^{2}$ are Lipschitz manifolds, J. Differential Geom. 39, 1 (1994), 65-101. MR1258915

[115] P. Tukia, Automorphic Quasimeromorphic Mappings for Torsionless Hyperbolic Groups. Ann. Acad. Sci. Fenn. Math. 10, 545-560, 1985. MR0802519

[116] C. Villani, Optimal Transport, Old and New, Grundlehren der mathematischen Wissenschaften 338, Springer, Berlin-Heidelberg, 2009. MR2459454

[117] A. Wald, Sur la courbure des surfaces, C. R. Acad. Sci. Paris, 201, 918-920, 1935. 
[118] A. Wald, Begreudeung einer koordinatenlosen Differentialgeometrie der Flächen. Ergebnisse e. Mathem. Kolloquims, First Series, 7, 2446, 1936.

[119] J. A. Wheeler, Geons, Phys. Rev. 97, 511-536, 1955. MR0067622

[120] J. A. Wheeler, Geometrodynamics and the Issue of the Final State, in Relativity, Groups and Topology, De Witt, C. and De Witt, B. (eds.), Gordon and Breach, 1964. MR0168332

[121] M. Zähle, Curvature Theory for Singular Sets in Euclidean Spaces, preprint, 2005.

EMIL SAUCAN

Max Planck Institute for Mathematics in the Sciences

INSELSTRASSE 2204103 LEIPZIG

GERMANY

AND

Department of Electrical EngineERing

TeChnion City, Haifa 32000

ISRAEL

E-mail address: Emil.Saucan@mis.mpg.de, semil@ee.technion.ac.il

Received 6 August 2015 